TRANSACTIONS OF THE

AMERICAN MATHEMATICAL SOCIETY

Volume 352, Number 3, Pages 1149-1169

S 0002-9947(99)02284-9

Article electronically published on March 10, 1999

\title{
ODD PRIMARY bo RESOLUTIONS AND CLASSIFICATION OF THE STABLE SUMMANDS OF STUNTED LENS SPACES
}

\author{
JESÚS GONZÁLEZ
}

\begin{abstract}
The classification of the stable homotopy types of stunted lens spaces and their stable summands can be obtained by proving the triviality of certain stable classes in the homotopy groups of these spaces. This is achieved in the 2-primary case by Davis and Mahowald using classical Adams spectral sequence techniques. We obtain the odd primary analogue using the corresponding Adams spectral sequence based at the spectrum representing odd primary connective $K$-theory. The methods allow us to answer a stronger problem: the determination of the smallest stunted space where such stable classes remain null homotopic. A technical problem prevents us from giving an answer in all situations; however, in a quantitative way, the number of cases missed is very small.
\end{abstract}

\section{INTRODUCTION}

Throughout this paper we let $p$ and $\mathbb{F}_{p}$ denote an odd prime and the field with $p$ elements respectively. Let $\mathbf{L}$ denote the infinite dimensional lens space $K\left(\mathbb{F}_{p}, 1\right)$ with its standard cell structure. Let $\mathbf{L}^{a}$ denote the $a^{\text {th }}$ dimensional skeleton of $\mathbf{L}$ and define the stunted lens space $\mathbf{L}_{a}^{b}$ to be the cofiber of the inclusion $\mathbf{L}^{a-1} \hookrightarrow \mathbf{L}^{b}$. There is a stable decomposition of $\mathbf{L}$ as a wedge of $p-1$ summands $\mathbf{B}(i), \quad i=$ $1, \cdots, p-1$ (spaces and decomposition are realized after one suspension), and this induces a corresponding stable decomposition for (the $p$ localization of) each $\mathbf{L}_{a}^{b}$ as a wedge of stunted spaces arising from the $\mathbf{B}(i)$ 's. This fact is used in [9] to obtain the complete classification of the homotopy types of stunted lens spectra from a partial classification of stunted $\mathbf{B}(i)$ spectra. On the other hand, it is observed that in this last problem we can restrict attention to a single value of $i$. The objective of this paper is to study the homotopy classification of stunted $\mathbf{B}(p-1)$ spectra.

Notation 1.1. Let $q$ stand for $2 p-2$ and let $\mathbf{B}$ denote $\mathbf{B}(p-1)$, which is a CW spectrum with cells in positive dimensions congruent with 0 and -1 modulo $q$. Let $\mathbf{B}^{n}$ denote the skeleton of $\mathbf{B}$ formed by the first $n$ cells. Thus $\mathbf{B}^{2 n}$ is a complex of dimension $n q$ whereas $\mathbf{B}^{2 n+1}$ is $(n+1) q-1$ dimensional. Let $\mathbf{B}_{m}^{n}$ denote the quotient space of $\mathbf{B}^{n}$ by $\mathbf{B}^{m-1}$. We will say that the top cell in $\mathbf{B}_{m}^{n}$ is integral if $n$ is odd and that its bottom cell is integral if $m$ is even.

The complete classification of stunted lens spectra is equivalent to the classification of stunted $\mathbf{B}$ spectra with at most one integral cell and it is obtained in [9];

Received by the editors November 18, 1994 and, in revised form, August 1, 1997.

1991 Mathematics Subject Classification. Primary 55P15; Secondary 55N20, 55P42, 55Q50, $55 \mathrm{~T} 15$.

(C)1999 American Mathematical Society 
therefore we will be mostly interested in stunted $\mathbf{B}$ spaces with both top and bottom integral cells. The starting point in the classification of such spectra is given by the following observations which are easily deduced from classical considerations with Adams's $J$-groups:

- If $i_{1} \equiv i_{2}\left(\bmod p^{j}\right)$, then $\mathbf{B}_{2 i_{1}}^{2 i_{1}+2 j+1} \simeq \mathbf{B}_{2 i_{2}}^{2 i_{2}+2 j+1}$.

- If $\mathbf{B}_{2 i_{1}}^{2 i_{1}+2 j+1} \simeq \mathbf{B}_{2 i_{2}}^{2 i_{2}+2 j+1}$, then $i_{1} \equiv i_{2}\left(\bmod p^{j-1}\right)$.

It is well known that for reducible or coreducible spectra, the optimal identifications are given by the first condition [9, Lemma 6.1]. Thus the problem consists in finding all possible identifications among the non-reducible nor coreducible spectra in

$$
\mathbf{B}_{2 i+2 t}^{2 i+2 t+2 j+1}, \quad t \in\left\{0, p^{j-1}, 2 p^{j-1}, \cdots,(p-1) p^{j-1}\right\} .
$$

Geometrical considerations reduce this problem to showing the triviality of certain stable homotopy classes which we now describe.

Definition 1.2. The $(i+1) q^{\text {th }}$ skeleton of $\Sigma \mathbf{B}_{2 i}^{2 i+2 l}$ is the wedge of two spheres of dimensions $i q+1$ and $(i+1) q$. Let $\iota: S^{(i+1) q} \hookrightarrow \Sigma \mathbf{B}_{2 i}^{2 i+2 l}$ denote the inclusion of the second sphere. Define the maps:

(a) $\theta_{l, i, j}$ to be the composite $S^{(i+j+1) q-1} \stackrel{\bar{\alpha}_{j}}{\longrightarrow} S^{(i+1) q} \stackrel{\iota}{\hookrightarrow} \Sigma \mathbf{B}_{2 i}^{2 i+2 l}$, where $\bar{\alpha}_{j}$ is a generator of the $p$-component of the image of $J$ in the stem $j q-1$,

(b) $\bar{\theta}_{l, i, j}$ to be the composite $S^{(i+j+1) q-1} \stackrel{\theta_{l, i, j}}{\longrightarrow} \Sigma \mathbf{B}_{2 i}^{2 i+2 l} \rightarrow \Sigma \mathbf{B}_{2 i+1}^{2 i+2 l}$, where the second map collapses to a point the bottom cell of $\Sigma \mathbf{B}_{2 i}^{2 i+2 l}$,

(c) $\vartheta_{l, i, j}$ to be the composite $S^{(i+j+1) q-1} \stackrel{\theta_{l, i, j}}{\longrightarrow} \Sigma \mathbf{B}_{2 i}^{2 i+2 l} \hookrightarrow \Sigma \mathbf{B}_{2 i}^{2 i+2 l+1}$, where the second map is the skeletal inclusion and

(d) $\bar{\vartheta}_{l, i, j}$ to be the composite $S^{(i+j+1) q-1} \stackrel{\vartheta_{l, i, j}}{\longrightarrow} \Sigma \mathbf{B}_{2 i}^{2 i+2 l+1} \rightarrow \Sigma \mathbf{B}_{2 i+1}^{2 i+2 l+1}$, where the second map collapses to a point the bottom cell of $\Sigma \mathbf{B}_{2 i}^{2 i+2 l+1}$.

We will denote $\theta_{l, i, j}$ simply by $\theta_{l}$ when $i$ and $j$ are clear from the context, and similarly for $\overline{\theta_{l}}, \vartheta_{l}$, and $\overline{\vartheta_{l}}$. Conditions implying stable identifications for the spaces in (1) are given by the following generalization to odd primes of the geometrical idea in [4].

Proposition 1.3 ([9, Proposition 6.3]). Let $t \equiv 0 \bmod p^{j-1}$. The spaces $\mathbf{B}_{2 i}^{2 i+2 j+1}$ and $\mathbf{B}_{2 i+2 t}^{2 i+2 t+2 j+1}$ are stably equivalent provided both $\theta_{j, i, j}$ and $\theta_{j, i^{\prime}, j}$ are stably null homotopic, where $i^{\prime}=p^{N}-i-t-j-1$ and $N \gg 0$.

In the 2-primary case, the classification of stunted projective spaces is achieved by showing that all potentially trivial " $\theta$-obstructions" indeed vanish. However, in the odd primary case we cannot expect to complete the classification by showing that all relevant " $\theta$-obstructions" always vanish. For instance, if $i=t=p^{j-1}$, then $\mathbf{B}_{2 i}^{2 i+2 j+1}$ and $\mathbf{B}_{2 i+2 t}^{2 i+2 t+2 j+1}$ have a "good chance" of being stably equivalent, but Proposition 4.1 implies that no stable equivalence can be obtained from Proposition 1.3. Note that in such case one of the spectra in (1) is S-coreducible. For these situations we need another set of obstructions to classify the remaining $p-1$ noncoreducible spectra. This is provided by maps of the form $\vartheta_{j, i, j}$. Indeed, a good understanding of such maps is required to obtain the complete classification of stunted B-spaces with top and bottom integral cells. This is formalized by the 
following result, whose hypothesis and conclusion are global, rather than the local nature of Proposition 1.3. The proof is given in Section 5 .

Proposition 1.4. Let $j$ be a positive integer. Assume $\vartheta_{j, i, j}$ is stably null homotopic whenever $\nu(i+j+1)<j$ (that is, whenever the target space of $\vartheta_{j, i, j}$ is not $S$ reducible). Then two spaces $\mathbf{B}_{2 a}^{2 a+2 j+1}$ and $\mathbf{B}_{2 b}^{2 b+2 j+1}$ which are neither S-reducible nor $S$-coreducible are stably equivalent if and only if $a \equiv b\left(\bmod p^{j-1}\right)$.

As an application of the calculations in this paper (Corollary 4.6, Theorem 5.3 and Proposition 5.6) we prove the triviality of all these " $\vartheta$-obstructions" for primes $p>7$ (see Remark 5.8), thus completing the classification of stunted B spaces for those primes. In fact, the techniques used enable us to identify the optimal situation for the stable triviality of maps in 1.2. More specifically, we study the following

Problem 1.5. For fixed $i$ and $j$, find the largest integer $l$ (if any) such that $\theta_{l}$ is stably essential. Same question for $\bar{\theta}_{l}, \vartheta_{l}$ and $\bar{\vartheta}_{l}$.

Our results concerning Problem 1.5 are as follows (see 4.1, 4.2, 4.3, 5.2, 5.3 and 5.6):

1. If $\nu(i+j+1) \geq j$, then for all $l$ the maps $\theta_{l}, \bar{\theta}_{l}, \vartheta_{l}$ and $\bar{\vartheta}_{l}$ are essential.

2. If $\nu(i+j+1)<j-1$, then in a wide range of cases (in a sense to be made precise in Section 5), the largest integer $l$ such that $\theta_{l}$ is essential is $l=\nu(i+j+1)+1$. The same value of $l$ applies in the case of the maps $\bar{\theta}_{l}, \vartheta_{l}$ and $\bar{\vartheta}_{l}$.

3. If $\nu(i+j+1)=j-1$, then $\theta_{j}$ and $\overline{\theta_{j}}$ are essential but $\vartheta_{j}$ and $\bar{\vartheta}_{j}$ are trivial.

We conjecture that part 2 remains true after removing the "wide range" restriction. Together with Proposition 1.4, this would finish up the classification of stunted B spectra.

We now give an overall description of the methods used. We show that a map is essential by checking that it has a nontrivial Hurewicz image under the $J$ spectrum. On the other hand, the homotopy triviality of $J$-trivial maps $S^{k} \rightarrow \Sigma \mathbf{B}_{a}^{b}$ in 1.2 is settled by showing that, in the classical Adams spectral sequence for $\Sigma \mathbf{B}_{a}^{b}$, such classes lie above a slanted line of $(t-s, s)$-slope close to $\frac{1}{p^{2}-p-1}$ and with the property that the only elements above the line, which survive to essential homotopy classes, must in fact be detected by the $J$-spectrum. For the sphere, this "vanishing" line is given in [8] and here we provide a slightly improved version (Theorem 5.1). For stunted $\mathbf{B}$ spectra and maps $\theta_{\ell}$ and $\bar{\theta}_{\ell}$, the line is settled in Theorems 5.2 and 5.3. The existence of such a vanishing line is established by using Mahowald's theory of bo-resolutions which, in general terms, gives a way of controlling the obstructions for lifting high-Adams filtration homotopy classes in $\pi_{*}(X)$ through a bo-resolution of certain spectra $X$. It is worth noticing that the techniques in [4] also make use of the fact that the maps under consideration have a high Adams filtration. In more detail, we find that the obstruction for the existence of a lifting to the second stage in the bo-resolution is given by $J$-homology groups, whereas a finite number of higher bo-obstructions can be controlled by the classical Adams filtration of the initial lifting. A technical problem arises at this point: one needs to get an initial lifting in the bo-resolution with a "large" Adams filtration. In general, there is a lower bound on the Adams filtration of this first lifting; however the bound is so rough that, in some cases, one could end up with no Adams filtration by the second 
stage. This problem appears in the case of bo-resolutions for the sphere spectrum as well as for stunted $\mathbf{B}$ spectra. It is however interesting to remark that, in the case of the sphere, one can in fact get an optimal control on the Adams filtration for the initial lifting to the second stage (compare parts (b) in Theorem 3.2 and its analogous results Theorems 3.3 and 3.4). At any rate, bo-resolution techniques give a way of lifting the maps in 1.2 through a finite number of stages in the boresolution of their targets. To show the maps are indeed trivial, that is, they lift all the way through the bo-resolution, we use general bounds for the bo-filtration of nontrivial homotopy classes over stunted $\mathbf{B}$ spectra.

The paper is arranged as follows: in Section 2 we identify and study the boregular cohomology for stunted $\mathbf{B}$ spectra. The information produces in Section 3 the two basic tools in the paper: on the one hand, a general criterion to lift homotopy classes through the bo-resolution of stunted B spectra (Theorems 3.3 and 3.4), and on the other, a vanishing line in the $E_{\infty}$ term of the bo-Adams spectral sequence (Proposition 3.5). The material in these two sections is based on calculations in [7] and represents the odd primary version of results in [5] and [13]. In Section 5 we apply these techniques in the study of Problem 1.5 and in particular in the classification of stunted $\mathbf{B}$ spectra. As pointed out above, we deduce the triviality of a number of the maps in 1.2 by lifting them through a bo-resolution of the relevant spectra. In Section 4 we describe the first bo-obstruction showing up in such liftings. The paper ends with a direct geometrical argument for proving the triviality of some of the maps in 1.2 not covered by the $K$-theoretic methods.

The material in this paper is part of the author's Ph.D. thesis written at the University of Rochester. It is a pleasure to gratefully acknowledge Professor Samuel Gitler for his advice during the preparation of this work.

\section{2. $\ell$-REgular homology for SPECTRA $\mathbf{B}_{2 a+1}^{2 b}$}

The spectrum representing connective $p$-local $K$-theory decomposes as a wedge of suspensions of the spectrum $B P\langle 1\rangle$ ([1], [2], [10]) which, from now on, will be denoted by $\ell$. In this section we describe following [13], the $\ell$-regular complex for stunted B spaces and derive the homology properties that lead to Mahowald's "Bounded Torsion Theorem" in the study of liftings through $\ell$-resolutions (cf. [5, Theorem 5.1] and [13, Theorem 7.1]). The definition of the $\ell$-regular complex $\mathcal{C}(X)$ is given in [7] and we will assume familiarity with the notation and calculations in that paper. In particular, we will only work with $p$-local connective finite type $\left(H \mathbb{F}_{p}, \ell\right)$-prime spectra [7, Definition 3.1]. For such a spectrum $X$, new spectra $X^{\langle k\rangle}(k \geq 0)$ are defined up to non natural homotopy equivalences by requiring $X^{\langle 0\rangle}$ to be a maximal wedge summand of $X$ having no split $\mathbb{F}_{p}$-Eilenberg Mac Lane summands and $X^{\langle 0\rangle} \leftarrow X^{\langle 1\rangle} \leftarrow X^{\langle 2\rangle} \leftarrow \cdots$ to be a minimal $\mathbb{F}_{p}$-Adams resolution of $X^{\langle 0\rangle}$. In this paper the spectrum $X^{\langle k\rangle}$ will be denoted by $X\langle k\rangle$ and the $\ell$-regular complex of $S^{0}\langle k\rangle$ by $\mathcal{C}(k)$.

Let $R$ denote the cofiber of the stable Kahn-Priddy map $\mathbf{B} \rightarrow S^{0}$ and let $R^{k}$ stand for the $k q+1$ dimensional skeleton of $R$ (recall $q=2 p-2$ ). Consider the maps

$$
R^{i}\langle k\rangle \rightarrow R^{i+j}\langle k\rangle \rightarrow R\langle k\rangle \rightarrow R\langle i+j+k\rangle \leftarrow S^{0}\langle i+j+k\rangle \leftarrow S^{0}\langle i+k\rangle
$$

where the first two maps are induced by skeletal inclusions, the third one is a lifting of the degree $p^{i+j}$ map on $R\langle k\rangle$, the fourth one is induced by the inclusion 


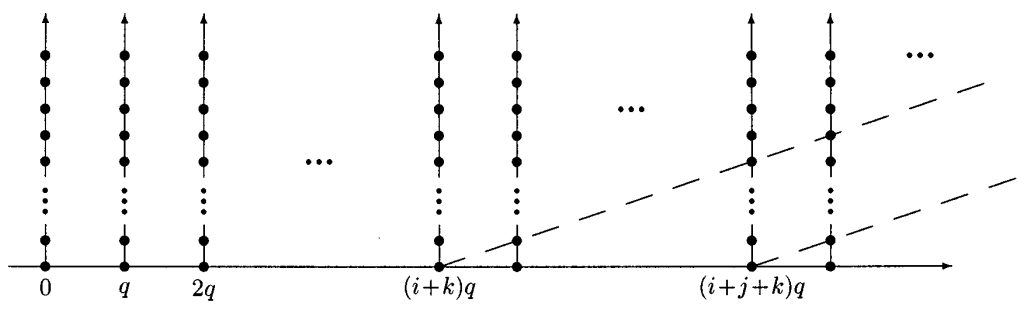

FiguRE 1

of the bottom cell and the last one is a lifting of the degree $p^{j}$ map on $S^{0}\langle i+k\rangle$. As remarked in [7, Section 5], all these maps can be computed in $\ell$-homology using the classical Adams spectral sequence together with a standard change-ofrings isomorphism. Thus the map in the middle induces isomorphisms, whereas the situation for both composites $R^{i}\langle k\rangle \rightarrow R^{i+j}\langle k\rangle \rightarrow R\langle k\rangle$ and $S^{0}\langle i+k\rangle \rightarrow$ $S^{0}\langle i+j+k\rangle \rightarrow R\langle i+j+k\rangle$ can be pictured in the Adams chart shown in Figure 1, where the image of $\ell_{*}\left(R^{i}\langle k\rangle\right)$ or $\ell_{*}\left(S^{0}\langle i+k\rangle\right)\left(\operatorname{resp} . \ell_{*}\left(R^{i+j}\langle k\rangle\right)\right.$ or $\left.\ell_{*}\left(S^{0}\langle i+j+k\rangle\right)\right)$ in $\ell_{*}(R\langle k\rangle)$ or $\ell_{*}(R\langle i+j+k\rangle)$ corresponds to the elements above the slanted line crossing the horizontal axis at $(i+k) q($ resp. $(i+j+k) q)$. Since all these are $\left(H \mathbb{F}_{p}, \ell\right)$-prime spectra, Mahowald's decomposition for $\left(\ell \wedge \bar{\ell}^{s}\right)\langle 0\rangle$ as a wedge of suspensions of spectra of the form $\ell\langle k\rangle[7$, Theorem 2.3] implies that the above observations extend to the corresponding $\ell$-regular complexes (for details see the notes after [7, Remark 3.4] or before [8, Proposition 3.4]). Thus, the middle map in (2) induces an isomorphism of complexes $\mathcal{C}(R\langle k\rangle) \simeq \mathcal{C}(R\langle i+j+k\rangle)$ under which $\mathcal{C}\left(R^{i+j}\langle k\rangle\right)$ is identified with $\mathcal{C}(i+j+k)$ and $\mathcal{C}\left(R^{i}\langle k\rangle\right)$ with $\mathcal{C}(i+k)$. In particular the first and last maps in (2) induce inclusions of $\ell$-regular complexes having isomorphic cokernels. From the bottom row of the diagram of cofibrations

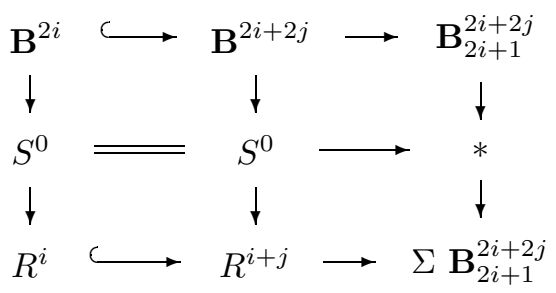

we see that the above isomorphism of cokernels can be rewritten as

$$
\mathcal{C}(i+j+k, i+k) \simeq \mathcal{C}\left(\Sigma \mathbf{B}_{2 i+1}^{2 i+2 j}\langle k\rangle\right)
$$

where $\mathcal{C}(i+j+k, i+k)$ denotes the cokernel of $\mathcal{C}(i+k) \hookrightarrow \mathcal{C}(i+j+k)$. This isomorphism is compatible under the inclusions induced by Adams projections.

The proofs of the following three propositions will be postponed until later in this section. Here the cohomology of $\mathcal{C}(i)$ and $\mathcal{C}(i+j, i)$ are denoted by $H(i)$ and $H(i+j, i)$ respectively; moreover $A$ will stand for either the chain map $\mathcal{C}(i+j, i) \rightarrow$ $\mathcal{C}(i+j-1, i-1)$ induced by Adams projection, or the corresponding homomorphism induced in cohomology groups.

Proposition 2.1. In cohomological degree $s \geq 3$, the groups $H(i+j, i)$ are $\mathbb{F}_{p^{-}}$ vector spaces and the maps $A: H(i+j, i) \rightarrow H(i+j-1, i-1)$ are trivial. 
By [7, Lemma 7.2] the map $A^{\nu(m)}: H^{2, q m}(i+\nu(m)) \rightarrow H^{2, q m}(i)$ is trivial and slight improvements on the power $\nu(m)$ can be obtained in particular cases. For the relative groups we have the following analogue.

Proposition 2.2. The composite $A^{r}: H^{2, q m}(i+j+r, i+r) \rightarrow H^{2, q m}(i+j, i)$ is trivial provided $r \geq 1$ and $r \geq \min \{m-i-j-1, \nu(m), \mu((p-1)(m+1-i))-1\}$. Here $\mu(n)$ denotes the integral part of the logarithm in base $p$ of $n$.

The groups $H^{1}(i+j, i)$ can be explicitly computed. They turn out to be cyclic; however orders are a bit irregular. We will only show the way in which they can be identified as subgroups of the $J$-homology of $\mathbf{B}_{2 i+1}^{2 i+2 j}$. In order to state the result, recall the $J$-spectrum is defined through a cofibration

$$
J \longrightarrow \ell \stackrel{\phi}{\longrightarrow} \Sigma^{q} \ell,
$$

where $\phi$ is given as follows: In Mahowald's splitting for $\ell \wedge \bar{\ell}$, the wedge summand with the lowest dimensional cell is $\Sigma^{q} \ell$. If $\ell \wedge \bar{\ell} \stackrel{\rho}{\longrightarrow} \Sigma^{q} \ell$ is the wedge projection, then $\phi$ is the composite $\ell \stackrel{p r}{\longrightarrow} \bar{\ell} \stackrel{\iota \wedge 1}{\longrightarrow} \ell \wedge \bar{\ell} \stackrel{\rho}{\longrightarrow} \Sigma^{q} \ell$, where $\iota: S^{0} \rightarrow \ell$ is the inclusion of the bottom cell and $p r$ fits in the cofibration $S^{0} \stackrel{\iota}{\longrightarrow} \ell \stackrel{p r}{\longrightarrow} \bar{\ell}$. For details see [3], [7], [11] or [12]. From [7, Lemma 3.3] the map $\ell \wedge \bar{\ell} \wedge X \stackrel{\rho \wedge 1}{\longrightarrow} \Sigma^{q} \ell \wedge X$ is trivial in homotopy classes arising from split $\mathbb{F}_{p}$-Eilenberg Mac Lane summands. Passing to the quotient we get an induced map

$$
\mathcal{C}^{1, *}(X)=\pi_{*}((\ell \wedge \bar{\ell} \wedge X)\langle 0\rangle) \stackrel{\rho}{\longrightarrow} \ell_{*-q}(X)
$$

which is identified with the projection onto the first summand in $\mathcal{C}^{1, *}(X)$. By [7, Theorem $3.6 \mathrm{~b}$ )] (or directly from the above description of $\phi$ ), the corresponding component of the differential $\mathcal{C}^{0, *}(X) \rightarrow \mathcal{C}^{1, *}(X)$ is induced by $\phi$, so that the composition of (5) with the connecting homomorphism $\ell_{*-q}(X) \rightarrow J_{*-1}(X)$ in (4) yields a well defined map $\tau: H^{1, *}(\mathcal{C}(X)) \rightarrow J_{*-1}(X)$.

Proposition 2.3. The group $H^{1, *}\left(\mathcal{C}\left(\Sigma \mathbf{B}_{2 i+1}^{2 i+2 j}\right)\right)$ is cyclic and injects into $J_{*-1}\left(\Sigma \mathbf{B}_{2 i+1}^{2 i+2 j}\right)$ under the map $\tau$ described above.

We now start with the proofs of Propositions 2.1, 2.2 and 2.3.

Proof of 2.1. The composite $\mathcal{C}(i+j, i) \rightarrow \mathcal{C}(i+j-1, i-1) \rightarrow \mathcal{C}(i+j, i)$ is multiplication by $p$ (as in (2), the second map is induced by liftings $S^{0}\langle i+j-1\rangle \rightarrow S^{0}\langle i+j\rangle$ and $S^{0}\langle i-1\rangle \rightarrow S^{0}\langle i\rangle$ of the degree $p$ maps in $S^{0}\langle i+j-1\rangle$ and $S^{0}\langle i-1\rangle$ ); therefore the first assertion is a consequence of the second one, which we now prove. Since the short exact sequences $0 \rightarrow \mathcal{C}(i) \rightarrow \mathcal{C}(i+j) \rightarrow \mathcal{C}(i+j, i) \rightarrow 0$ and $0 \rightarrow \mathcal{C}(i-1) \rightarrow \mathcal{C}(i+j-1) \rightarrow \mathcal{C}(i+j-1, i-1) \rightarrow 0$ are compatible under Adams projections, we have the commutative diagram with exact rows

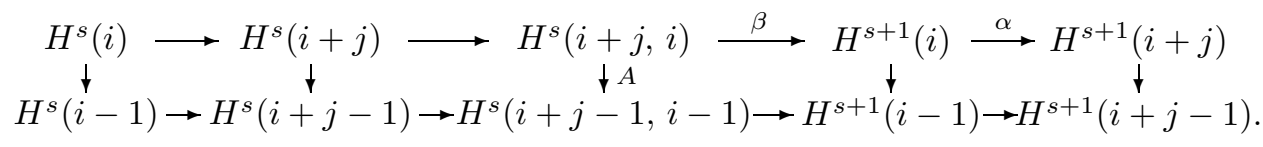

By [7, Lemma 7.1], the second vertical map is the zero morphism, so that the triviality of $A$ will follow by showing that every element in Ker $\alpha$ has a preimage under $\beta$ in Ker $A$. This is proved by direct calculation. Recall from [7, Corollary 
6.11 (b)] that in homological degree at least $3, H^{*}(i)$ has two kinds of representative cycles:

1. $\frac{1}{p} d D_{0} w_{i}^{m} h^{I}$ with $h^{I}$ admissible, $\nu(m)<k=k(I)$ and $i+\nu(I) \leq m<i+$ $\nu(I)+\nu\left(p^{\nu(m)+1}\right.$ !) (the inequality $i+\nu(I) \leq m$ rules out the differential $\frac{1}{p} w_{i}^{m} \partial h^{I} \rightarrow \frac{1}{p} w_{i}^{m-p^{\nu(m)}} \partial\left(u_{\nu(m)} h^{I}\right)$ in the weight spectral sequence for $\left.\mathcal{C}(i)\right)$, and

2. $\frac{1}{p} d \bar{D}_{0} w_{i}^{m} h^{I}$ with $h^{I}$ admissible starting in the form $h^{I}=u_{k} u_{k+d} \cdots, \nu(m)=$ $k, c(m)=p-1$ and $i+\nu(I) \leq m<i+\nu(I)+p^{k+1}-1$ (so that the differential $\frac{1}{p} w_{i}^{m} \partial h^{I} \rightarrow \frac{1}{p} w_{i}^{m-(p-1) p^{k}} \partial g^{I^{\prime}}$ in the weight spectral sequence for $\mathcal{C}(i)$ is ruled out by the inequality $i+\nu(I) \leq m)$.

We will consider the case where the class in $\operatorname{Ker} \alpha$ is represented by a cycle of type 2 (the situation for type 1 cycles is easier and represents the analogous situation at the prime 2 in [13, Proposition 7.4]). Let $x=\frac{1}{p} d \bar{D}_{0} w_{i}^{m} h^{I} \in \mathcal{C}(i)$ be a representative cycle for an element in $\operatorname{Ker} \alpha$, so that $h^{I}=u_{k} u_{k+d} \cdots$ is admissible, $\nu(m)=k, c(m)=p-1$ and

$$
i+\nu(I) \leq m<i+\nu(I)+p^{k+1}-1 .
$$

The assumption $x \in \operatorname{Ker} \alpha$ means

$$
m<i+j+\nu(I)
$$

(so that the differential ruled out in $\mathcal{C}(i)$ does hold in $\mathcal{C}(i+j)$ ). Then $z=$ $\frac{1}{p} \bar{D}_{0} w_{i+j}^{m} h^{I}$ lies in $\mathcal{C}(i+j)$ and projects into the quotient $\mathcal{C}(i+j, i)$ to a cycle whose homology class hits $x$ under $\beta$. The proof is completed by verifying that, as an element in $\mathcal{C}(i+j-1), A(z)$ is homologous to an element in $\mathcal{C}(i-1)$. By [7, Definition $5.14(\mathrm{~d})$ ]

$$
A(z)=\sum_{\lambda=0}^{p-2} u_{\lambda} D_{0}^{\prime} w_{i+j-1}^{m-\lambda p^{k}}\left[\vartheta_{(\lambda+1) p^{k}}\right] h^{I-\Delta_{k}}+w_{i+j-1}^{m-(p-1) p^{k}}\left(D_{00} u_{k+1}\right) h^{I-\Delta_{k}},
$$

where each $u_{\lambda}$ is a unit in the $p$-localized integers. Direct calculations show that

(i). (6) implies $w_{i-1}^{m-(p-1) p^{k}}\left(D_{00} u_{k+1}\right) h^{I-\Delta_{k}} \in \mathcal{C}(i-1)$.

(ii). (7) implies $w_{i+j-1}^{m-\lambda p^{k}}\left[\vartheta_{(\lambda+1) p^{k}}\right] h^{I-\Delta_{k}} \in \mathcal{C}(i+j-1), \quad 0 \leq \lambda \leq p-2$.

(iii). (6) implies $\sum_{\lambda=0}^{p-2} u_{\lambda} D_{1}^{\prime} w_{i-1}^{m-\lambda p^{k}}\left[\vartheta_{(\lambda+1) p^{k}}\right] h^{I-\Delta_{k}} \in \mathcal{C}(i-1)$.

Since $d=D_{0}^{\prime}+D_{1}^{\prime}$, (ii) and (iii) imply that the summation in the above formula for $A(z)$ is homologous in $\mathcal{C}(i+j-1)$ to an element in $\mathcal{C}(i-1)$, then (i) shows it is also true for the whole $A(z)$ as desired.

We will only comment on (iii) - partially to illustrate the way one handles the cancellations in $[7,(34)]$. In weight filtration at most $p^{k+1}+W F\left(h^{I-\Delta_{k}}\right)$ (where $W F\left(h^{I-\Delta_{k}}\right)$ is the weight filtration of $h^{I-\Delta_{k}}$ ) the components of the sum in (iii) reduce to a linear combination of elements of the form

$$
w_{i-1}^{m-(p-1) p^{k}}\left[\vartheta_{(p-\lambda-1) p^{k}} \mid \vartheta_{(\lambda+1) p^{k}}\right] h^{I-\Delta_{k}},
$$

for $0 \leq \lambda \leq p-2$ (cf. [7, (34)]), each one lying in $\mathcal{C}(i-1)$ by direct calculation as in (i). On the other hand, expanding the sum in (iii), one finds that the components in weight $>p^{k+1}+W F\left(h^{I-\Delta_{k}}\right)$ are linear combinations of elements of the form 
$w_{i-1}^{m-\lambda p^{k}-r}\left[\vartheta_{r} \mid \vartheta_{(\lambda+1) p^{k}}\right] h^{I-\Delta_{k}}$ with $r+(\lambda+1) p^{k}>p^{k+1}$ and $0 \leq \lambda \leq p-2$, but this last restriction together with (6) implies that such elements lie in $\mathcal{C}(i-1)$.

Proof of 2.2. With the obvious modifications the main body of the previous proof applies in this case (since $s=2$, only cycles of type 1 have to be considered) and this time we must show in addition that under the present hypothesis, the composite $H^{2, q m}(i+j+r) \stackrel{A^{r}}{\longrightarrow} H^{2, q m}(i+j) \longrightarrow H^{2, q m}(i+j, i)$ is the trivial map. The case $r \geq \nu(m)$ follows at once from [7, Lemma 7.2], whereas $H^{2, q m}(i+j+r)=0$ for $r \geq m-i-j-1$ in view of [7, Corollary 6.11 (a)]. The result for $r \geq$ $\mu((p-1)(m+1-i))-1$ is also a consequence of [7], but we give more details. Recall from [7, Corollary 6.11 (a)] that $H^{2, q m}(i)=0$ for $i \geq m-1$, whereas for $i<m-1$ the group is cyclic with generators $G_{t}($ for $\mu((p-1)(m+1-i))-1 \leq$ $t<\min \{\nu(m), m-i-1\})$ and relations $p G_{t}=G_{t+1}$. Each $G_{t}$ is represented by the cycle $\frac{1}{p^{\nu(m)-t}} d\left(\sum_{r<p^{t+1}}\left(\left(\begin{array}{c}m \\ r\end{array}\right)\right) w_{i}^{m-r}\left[\vartheta_{r}\right]\right)$ (cf. [7, Remark 5.22 (iii)]). It follows that under the map $H^{2, q m}(i) \rightarrow H^{2, q m}(i+j)$ each element $G_{t}$ maps to the corresponding $G_{t}$, whereas $G_{t}$ is sent to $G_{t+r}$ under the Adams projection $H^{2, q m}(i+j+r) \rightarrow H^{2, q m}(i+j)$. Thus the condition $r \geq \mu((p-1)(m+1-i))-1$ implies that the image of the latter map is contained in the image of the former one, implying the result.

Proof of 2.3. Recall the groups $\ell_{*}\left(\Sigma \mathbf{B}_{2 i+1}^{2 i+2 j}\right)$ are given by a chart of the form shown in Figure 2 (where $j=3$ ). Moreover, the group $J_{q m-1}\left(\Sigma \mathbf{B}_{2 i+1}^{2 i+2 j}\right)$ can be computed as the cokernel of the map $\ell_{q m}\left(\Sigma \mathbf{B}_{2 i+1}^{2 i+2 j}\right) \stackrel{\phi}{\longrightarrow} \ell_{q(m-1)}\left(\Sigma \mathbf{B}_{2 i+1}^{2 i+2 j}\right)$ which sends the generator in $\ell_{q m}\left(\Sigma \mathbf{B}_{2 i+1}^{2 i+2 j}\right)$ into the $p^{\nu(m)+\epsilon}$-multiple of the generator in $\ell_{q(m-1)}\left(\Sigma \mathbf{B}_{2 i+1}^{2 i+2 j}\right)$, where $\epsilon=1$ if $m>i+j$ and $\epsilon=0$ otherwise (see for instance [14, Section 3]). On the other hand, the group $H^{1, q m}(i+j, i)$ is calculated with the exact sequence

$$
H^{1, q m}(i) \stackrel{\alpha_{1}}{\longrightarrow} H^{1, q m}(i+j) \stackrel{\beta}{\longrightarrow} H^{1, q m}(i+j, i) \stackrel{\delta}{\longrightarrow} H^{2, q m}(i) \stackrel{\alpha_{2}}{\longrightarrow} H^{2, q m}(i+j)
$$

and we will show that the middle group is cyclic and any element of order $p$ on it is sent nontrivially to $J_{q m-1}\left(\Sigma \mathbf{B}_{2 i+1}^{2 i+2 j}\right)$ under $\tau$. The calculations are based on results in [7], specifically on Remark 5.22 (iii), Example 6.10 and Corollary 6.11 (a).

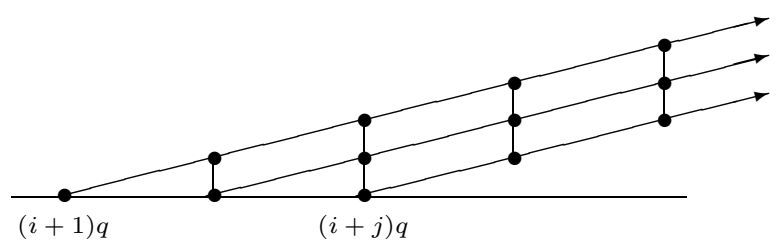

FiguRE 2 
First we show that if Coker $\alpha_{1} \neq 0$, the element of order $p$ it produces in $H^{1, q m}(i+j, i)$ maps nontrivially to $J_{q m-1}\left(\Sigma \mathbf{B}_{2 i+1}^{2 i+2 j}\right)$. Indeed, in this case we must have

(a) $i+j<m \quad\left(\right.$ since $\left.H^{1, q m}(i+j) \neq 0\right)$.

(b) $m-i-1-\nu(m)>0 \quad$ (otherwise $\alpha_{1}$ would be onto) so that $H^{1, q m}(i)=$ $\mathbf{Z} / p^{\nu(m)+1}$ with generator represented by $d\left(p^{m-i-1-\nu(m)} w_{i}^{m}\right)$.

(c) $H^{1, q m}(i+j)=\mathbf{Z} / p^{\min \{m-i-j, \nu(m)+1\}}$ with generator represented by the element $d\left(p^{\max \{0, m-i-j-1-\nu(m)\}} w_{i+j}^{m}\right)$. In particular, the class of order $p$ is represented by $d\left(p^{m-i-j-1} w_{i+j}^{m}\right)$.

Thus the class of order $p$ in Coker $\alpha_{1}$ is represented by

$$
d\left(p^{\min \{m-i-j-1, m-i-2-\nu(m)\}} w_{i+j}^{m}\right),
$$

which up to a unit and modulo classes of weight filtration larger than 1 , agrees with $p^{\min \{m-i-j-1+\nu(m), m-i-2\}} w_{i+j}^{m-1} u_{0}$. Using the isomorphism (3), this maps under $\rho$ of (5) to $p^{m-i-j-1+\min \{\nu(m), j-1\}} w^{m-1}$ in $\ell_{q(m-1)}\left(\Sigma \mathbf{B}_{2 i+1}^{2 i+2 j}\right)$, which by the previous description of the relevant $J$ groups, maps in turn nontrivially to $J_{q m-1}\left(\Sigma \mathbf{B}_{2 i+1}^{2 i+2 j}\right)$.

Next we show that if Coker $\alpha_{1} \neq 0 \neq \operatorname{Ker} \alpha_{2}$, the extension they define is nontrivial. Indeed, in addition to (a), (b) and (c) we now have

(d) $\mu((p-1)(m+1-i))-1<\nu(m)$ and $i<m-1 \quad$ (since $\left.H^{2, q m}(i) \neq 0\right)$.

(e) $\nu(m)>m-i-j-1 \quad$ (otherwise $\alpha_{2}$ would be monic).

Then (b) and (e) imply that the element of order $p$ in $\operatorname{Ker} \alpha_{2}$ is $G_{\nu(m)-1}$, which as above is represented by the cycle

$$
\frac{1}{p} d\left(\sum_{r<p^{\nu(m)}}\left(\left(\begin{array}{c}
m \\
r
\end{array}\right)\right) w_{i}^{m-r}\left[\vartheta_{r}\right]\right) \in \mathcal{C}(i) .
$$

Let $z=\sum_{r<p^{\nu(m)}} \frac{1}{p}\left(\left(\begin{array}{c}m \\ r\end{array}\right)\right) w_{i+j}^{m-r}\left[\vartheta_{r}\right]$. For $1 \leq r<p^{\nu(m)}$, [7, Lemma 4.8 (c)] gives $\nu\left(\begin{array}{l}m \\ r\end{array}\right)=\nu(m)-\nu(r)$; therefore (e) implies that each summand of $z$ lies in $\mathcal{C}(i+j)$. By construction $z$ projects to a cycle in $\mathcal{C}(i+j, i)$ whose homology class hits $G_{\nu(m)-1}$ under $\delta$. On the other hand, (c) and (e) imply that the generator in $H^{1, q m}(i+j)$ is represented by $d\left(w_{i+j}^{m}\right)$ and it suffices to verify that this element differs from $p z$ by an element in $\mathcal{C}(i)$. In other words, we have to show that $\sum_{r \geq p^{\nu(m)}}\left(\left(\begin{array}{c}m \\ r\end{array}\right)\right) w_{i}^{m-r}\left[\vartheta_{r}\right] \in$ $\mathcal{C}(i)$, or equivalently that $m-r \leq \nu\left(\begin{array}{c}m \\ r\end{array}\right)+i+\nu(r !)$ for $r \geq p^{\nu(m)}$ (cf. [7, (36) or (38)]). We will verify that in fact $m-p^{\nu(m)}<i+\nu\left(p^{\nu(m)}\right.$ !). Indeed, another way of stating (d) is $(p-1)(m+1-i)<p^{\nu(m)+1}$, or equivalently $m+1-i \leq \frac{p^{\nu(m)+1}-1}{p-1}=$ $\nu\left(p^{\nu(m)+1} !\right)$, as desired.

Finally we show that if Coker $\alpha_{1}=0$ but $\operatorname{Ker} \alpha_{2} \neq 0$, the element of order $p$ produced in $H^{1, q m}(i+j, i)$ maps nontrivially to $J_{q m-1}\left(\Sigma \mathbf{B}_{2 i+1}^{2 i+2 j}\right)$. Indeed, this time we must have in addition to (d) and (e) either one of the following:

(f) $i+j \geq m$ (so that $H^{1, q m}(i+j)=0$ ), or

(g) $\quad \nu(m) \geq m-i-1$ (so that $\alpha_{1}$ is onto).

If (g) holds, the class of order $p$ in $\operatorname{Ker} \alpha_{2}$ is $G_{m-i-2}$, which as an element of $\mathcal{C}(i)$ is represented by the cycle $\frac{1}{p^{\nu(m)-m+i+2}} d\left(\sum_{r<p^{m-i-1}}\left(\left(\begin{array}{c}m \\ r\end{array}\right)\right) w_{i}^{m-r}\left[\vartheta_{r}\right]\right)$. As above, the element $z=\frac{1}{p^{\nu(m)-m+i+2}} \sum_{r<p^{m-i-1}}\left(\left(\begin{array}{c}m \\ r\end{array}\right)\right) w_{i+j}^{m-r}\left[\vartheta_{r}\right]$ lies in $\mathcal{C}(i+j)$ and 
projects to a cycle in $\mathcal{C}(i+j, i)$ whose homology class hits $G_{m-i-2}$ under the boundary homomorphism $\delta$. We have to see that the resulting homology class in $H^{1, q m}(i+j, i)$ maps nontrivially into $J_{q m-1}\left(\Sigma \mathbf{B}_{2 i+1}^{2 i+2 j}\right)$ under $\tau$. Up to units, the representative cycle maps under $\rho$ of $(5)$ to $p^{m-i-2} w^{m-1}$ in $\ell_{q(m-1)}\left(\Sigma \mathbf{B}_{2 i+1}^{2 i+2 j}\right)$ (the nontrivial element of order $p$ ) but $(\mathrm{g})$ implies that the last group is isomorphic to $J_{q m-1}\left(\Sigma \mathbf{B}_{2 i+1}^{2 i+2 j}\right)$ (whether or not (f) holds). On the other hand, if (f) holds but (g) does not, then the element of order $p$ in $\operatorname{Ker} \alpha_{2}$ is $G_{\nu(m)-1}(\nu(m)>0$ by $(\mathrm{d}))$, and as before the element it produces in $H^{1, q m}(i+j, i)$ maps to $p^{\nu(m)-1} w^{m-1} \in$ $\ell_{q(m-1)}\left(\Sigma \mathbf{B}_{2 i+1}^{2 i+2 j}\right)$, which in turn projects nontrivially to $J_{q m-1}\left(\Sigma \mathbf{B}_{2 i+1}^{2 i+2 j}\right)$.

Let $S^{i q+1} \hookrightarrow \Sigma \mathbf{B}_{2 i}^{2 i+2 j}$ be the inclusion of the bottom cell. Since the $\ell$-regular complexes $\mathcal{C}\left(S^{i q+1}\right)$ and $\mathcal{C}\left(\Sigma \mathbf{B}_{2 i+1}^{2 i+2 j}\right)$ are respectively concentrated in total degrees congruent with 1 and 0 modulo $q$, the cofiber sequence $S^{i q+1} \hookrightarrow \Sigma \mathbf{B}_{2 i}^{2 i+2 j} \rightarrow$ $\Sigma \mathbf{B}_{2 i+1}^{2 i+2 j}$ yields an isomorphism of complexes

$$
\mathcal{C}\left(\Sigma \mathbf{B}_{2 i}^{2 i+2 j}\right) \simeq \mathcal{C}\left(S^{i q+1}\right) \oplus \mathcal{C}\left(\Sigma \mathbf{B}_{2 i+1}^{2 i+2 j}\right) .
$$

In particular 2.1 and 2.2 together with their analogous results for the sphere spectrum (Lemmas 7.1 and 7.2 in [7]) yield the following:

Proposition 2.4. In cohomological degrees $s \geq 2$ and total degrees $t$, the $r$-fold iterated composition $\Sigma \mathbf{B}_{2 i}^{2 i+2 j}\langle i\rangle \rightarrow \Sigma \mathbf{B}_{2 i}^{2 i+2 j}\langle i-1\rangle \rightarrow \cdots \rightarrow \Sigma \mathbf{B}_{2 i}^{2 i+2 j}\langle i-r\rangle$ of the Adams projections in a minimal $\mathbb{F}_{p}$-resolution of $\Sigma \mathbf{B}_{2 i}^{2 i+2 j}$ induces the trivial map $H^{s t}\left(\mathcal{C}\left(\Sigma \mathbf{B}_{2 i}^{2 i+2 j}\langle i\rangle\right)\right) \rightarrow H^{s t}\left(\mathcal{C}\left(\Sigma \mathbf{B}_{2 i}^{2 i+2 j}\langle i-r\rangle\right)\right)$ provided

a) $s \geq 3$ and $r=1$,

b) $s=2, t=q m+1$ and $r=\nu(m-i)$,

c) $s=2, t=q m$ and $r=\max \{1, \nu(m)\}$.

From the definition of the map $\tau: H^{1, *}(\mathcal{C}(X)) \rightarrow J_{*-1}(X)$ we have a commutative diagram

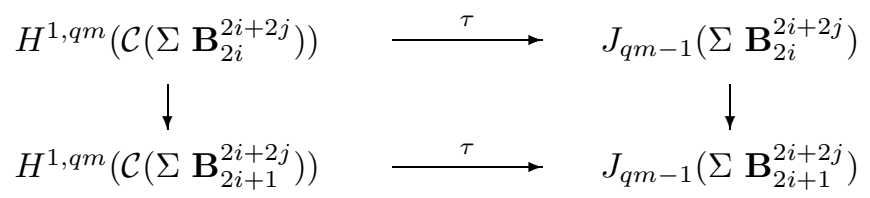

where both vertical maps are induced by the collapsing $\Sigma \mathbf{B}_{2 i}^{2 i+2 j} \rightarrow \Sigma \mathbf{B}_{2 i+1}^{2 i+2 j}$. In 4.1 we observe that the vertical map on the right is an isomorphism; therefore the next result follows from (8) and 2.3.

Proposition 2.5. $\tau: H^{1, q m}\left(\mathcal{C}\left(\Sigma \mathbf{B}_{2 i}^{2 i+2 j}\right)\right) \rightarrow J_{q m-1}\left(\Sigma \mathbf{B}_{2 i}^{2 i+2 j}\right)$ is a monomorphism.

\section{Liftings THROUGH $\ell$-RESOLUTIONS}

Mahowald's bounded torsion theorem [13, Theorem 7.1] depends on the fact that, for a number of spectra, $\ell$-obstructions for liftings through an $\ell$-resolution behave like $\mathbb{F}_{p}$-obstructions: they vanish provided one has enough classical Adams filtration. Such a result follows from detailed calculations like those in the previous section, together with the next mild generalization of [5, Lemma 5.8]. Recall the 
Adams filtration of a map $g: X \rightarrow Y$, denoted by $A F(g)$, is the maximum length $l$ of factorizations of $g$ in the form $X=X_{0} \stackrel{g_{1}}{\longrightarrow} X_{1} \stackrel{g_{2}}{\longrightarrow} X_{2} \stackrel{g_{3}}{\longrightarrow} \cdots \stackrel{g_{l}}{\longrightarrow} X_{l}=Y$, with each $g_{i}$ inducing the trivial morphism in $\bmod p$ homology.

Lemma 3.1. Let $X \stackrel{f}{\rightarrow} Y \stackrel{g}{\rightarrow} Z$ be a cofibration of spectra and assume that the classical $\mathbb{F}_{p}$-Adams spectral sequence computing $\pi_{*}(Z)$ collapses from its second term. If $\alpha \in \pi_{n}(Y)$ maps trivially under $g_{*}$, then there exists $\beta \in \pi_{n}(X)$ with $f_{*}(\beta)=\alpha$ and $A F(\beta) \geq A F(\alpha)-1$.

Proof. As is well known, from any $\mathbb{F}_{p}$-resolution $\cdots \stackrel{p_{3}}{\longrightarrow} Z_{2} \stackrel{p_{2}}{\longrightarrow} Z_{1} \stackrel{p_{1}}{\longrightarrow} Z_{0}=Z$ of

$Z$, we can extract a minimal $\mathbb{F}_{p}$-resolution $\cdots \stackrel{p_{3}^{\prime}}{\longrightarrow} Z_{2}^{\prime} \stackrel{p_{2}^{\prime}}{\longrightarrow} Z_{1}^{\prime} \stackrel{p_{1}^{\prime}}{\longrightarrow} Z_{0}^{\prime}=Z$ so that each $Z_{i}^{\prime}$ is a wedge summand of $Z_{i}$ having as cosummand a wedge of $\mathbb{F}_{p}$-Eilenberg Mac Lane spectra, with $p_{i}^{\prime}$ the corresponding component of $p_{i}$, and such that every homotopy class arising from an $\mathbb{F}_{p}$-Eilenberg Mac Lane summand maps trivially under $p_{i}$ (see for instance [8, Lemma 3.1] or [7, Remark 3.4]). As observed in [7, Remark 3.2], each $p_{i}^{\prime}$ must induce an injective homomorphism in homotopy groups. With these observations in mind, the proof of [5, Lemma 5.8] applies in our case. For completeness we supply the details. Letting $\cdots \stackrel{\pi_{3}}{\longrightarrow} A_{2} \stackrel{\pi_{2}}{\longrightarrow} A_{1} \stackrel{\pi_{1}}{\longrightarrow} A_{0}=S^{0}$ denote any $\mathbb{F}_{p}$-Adams resolution of $S^{0}$, choose $\alpha_{a} \in \pi_{n}\left(A_{a} \wedge Y\right)$ a lifting of $\alpha \in$ $\pi_{n}(Y)$, where $a=A F(\alpha)$. Then the observations above applied to the resolution $\ldots \stackrel{\pi_{3} \wedge 1}{\longrightarrow} A_{2} \wedge Z \stackrel{\pi_{2} \wedge 1}{\longrightarrow} A_{1} \wedge Z \stackrel{\pi_{1} \wedge 1}{\longrightarrow} A_{0} \wedge Z=Z$ imply that $\alpha_{a}$ maps trivially into $\pi_{n}\left(A_{a-1} \wedge Z\right)$ in the diagram

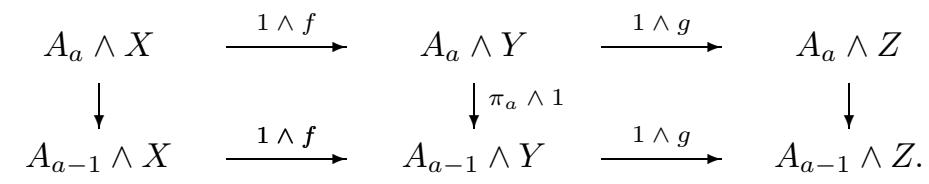

Therefore there exists $\beta_{a-1} \in \pi_{n}\left(A_{a-1} \wedge X\right)$ with $(1 \wedge f)_{*}\left(\beta_{a-1}\right)=\left(\pi_{a} \wedge 1\right)\left(\alpha_{a}\right)$. The image of $\beta_{a-1}$ under the composite $\pi_{n}\left(A_{a} \wedge X\right) \rightarrow \pi_{n}\left(A_{a-1} \wedge X\right) \rightarrow \cdots \rightarrow \pi_{n}(X)$ yields the required class $\beta$.

The basic result about liftings through an $\ell$-resolution of the sphere spectrum is given by the following odd primary version of [5, Theorem 5.1].

Theorem 3.2. Let $S^{0} \stackrel{p_{1}}{\longleftarrow} S_{1} \stackrel{p_{2}}{\longleftarrow} S_{2} \stackrel{p_{3}}{\longleftarrow} \cdots$ be the standard $\ell$-resolution of the sphere spectrum and suppose given a map $S^{n} \stackrel{f_{s}}{\longrightarrow} S_{s}$.

(a) In the case $s=0$ and $n>0, f_{0}$ can always be lifted to a map $f_{1}: S^{n} \rightarrow S_{1}$ with $A F\left(f_{1}\right) \geq A F\left(f_{0}\right)-1$.

(b) In the case $s=1$ assume $A F\left(f_{1}\right) \geq 1$ and that $S^{n} \stackrel{f_{1}}{\longrightarrow} S_{1} \stackrel{p_{1}}{\longrightarrow} S^{0} \stackrel{j}{\longrightarrow} J$ composes trivially, where $j: S^{0} \rightarrow J$ is the inclusion of the bottom cell. Then there is a map $S^{n} \stackrel{f_{2}}{\longrightarrow} S_{2}$ such that $p_{1} \circ p_{2} \circ f_{2}=p_{1} \circ f_{1}$ and with $A F\left(f_{2}\right) \geq$ $A F\left(f_{1}\right)-1$.

(c) In the case $s=2$ assume $A F\left(f_{2}\right) \geq \epsilon_{2}$, where $\epsilon_{2}=1$ if $n+2 \not \equiv 0(\bmod q)$ and $\epsilon_{2}=1+\nu(n+2)$ otherwise. Then there is a map $S^{n} \stackrel{f_{3}}{\longrightarrow} S_{3}$ such that $p_{2} \circ p_{3} \circ f_{3}=p_{2} \circ f_{2}$ and with $A F\left(f_{3}\right) \geq A F\left(f_{2}\right)-\epsilon_{2}$. 
(d) In the case $s \geq 3$ assume $A F\left(f_{s}\right) \geq \epsilon_{s}$ where $\epsilon_{s}=1$ if $n+s \not \equiv 0(\bmod q)$ and $\epsilon_{s}=2$ otherwise. Then there is a map $S^{n} \stackrel{f_{s+1}}{\longrightarrow} S_{s+1}$ such that $p_{s} \circ p_{s+1} \circ f_{s+1}=$ $p_{s} \circ f_{s}$ and with $A F\left(f_{s+1}\right) \geq A F\left(f_{s}\right)-\epsilon_{s}$.

This result appears as Theorem 7.5 in [7] where it is proved in detail. For our applications we will need the corresponding analysis of liftings through an $\ell$ resolution of stunted $\mathbf{B}$ spectra.

Theorem 3.3. Let $\Sigma \mathbf{B}_{2 i+1}^{2 i+2 j} \stackrel{p_{1}}{\longleftarrow} S_{1} \stackrel{p_{2}}{\longleftarrow} S_{2} \stackrel{p_{3}}{\longleftarrow} \cdots$ be the standard $\ell$-resolution of $\Sigma \mathbf{B}_{2 i+1}^{2 i+2 j}$ and suppose given a map $S^{n} \stackrel{f_{s}}{\longrightarrow} S_{s}$.

(a) In the case $s=0$ assume $S^{n} \stackrel{f_{0}}{\longrightarrow} \Sigma \mathbf{B}_{2 i+1}^{2 i+2 j} \stackrel{\iota \wedge 1}{\longrightarrow} \ell \wedge \mathbf{B}_{2 i+1}^{2 i+2 j}$ composes trivially; then $f_{0}$ can be lifted to a map $f_{1}: S^{n} \rightarrow S_{1}$ with $A F\left(f_{1}\right) \geq A F\left(f_{0}\right)-1$.

(b) In the case $s=1$ assume $A F\left(f_{1}\right) \geq 1$ and that $S^{n} \stackrel{f_{1}}{\longrightarrow} S_{1} \stackrel{p_{1}}{\longrightarrow} \Sigma \mathbf{B}_{2 i+1}^{2 i+2 j} \stackrel{j \wedge 1}{\longrightarrow}$ $J \wedge \Sigma \mathbf{B}_{2 i+1}^{2 i+2 j}$ composes trivially. If $n+1 \equiv 0(\bmod q)$, suppose in addition that $\frac{n+1}{q}>i+j$. Then there is a map $S^{n} \stackrel{f_{2}}{\longrightarrow} S_{2}$ such that $p_{1} \circ p_{2} \circ f_{2}=p_{1} \circ f_{1}$ and with

(i) $A F\left(f_{2}\right) \geq A F\left(f_{1}\right)-1$ in case $n+1 \not \equiv 0(\bmod q)$, or $n+1=q m$ with $\nu(m) \geq j-1$.

(ii) $A F\left(f_{2}\right) \geq A F\left(f_{1}\right)-\nu(m)-1$ in case $n+1=q m$ with $\nu(m)<j-1$.

(c) In the case $s=2$ assume one of the following

(i) $n+2 \not \equiv 0(\bmod q)$ and $A F\left(f_{2}\right) \geq 1$.

(ii) $n+2=q m$ and $A F\left(f_{2}\right) \geq \max \{2, \nu(m)+1\}$.

(iii) $n+2=q m$ and $A F\left(f_{2}\right) \geq \max \{2, m-i-j-1\}$.

(iv) $n+2=q m$ and $A F\left(f_{2}\right) \geq \max \{2, \mu((p-1)(m+1-i))\} \quad$ ( $\mu$ as in 2.2).

Then there is a map $S^{n} \stackrel{f_{3}}{\longrightarrow} S_{3}$ such that $p_{2} \circ p_{3} \circ f_{3}=p_{2} \circ f_{2}$ and with

(i) $A F\left(f_{3}\right) \geq A F\left(f_{2}\right)-1$,

(ii) $A F\left(f_{3}\right) \geq A F\left(f_{2}\right)-\max \{2, \nu(m)+1\}$,

(iii) $A F\left(f_{3}\right) \geq A F\left(f_{2}\right)-2$,

(iv) $A F\left(f_{3}\right) \geq A F\left(f_{2}\right)-\max \{2, \mu((p-1)(m+1-i))\}$, respectively.

(d) In the case $s \geq 3$ assume $A F\left(f_{s}\right) \geq \epsilon_{s}$, where $\epsilon_{s}=1$ if $n+s \not \equiv 0(\bmod q)$ and $\epsilon_{s}=2$ otherwise. Then there is a map $S^{n} \stackrel{f_{s+1}}{\longrightarrow} S_{s+1}$ such that $p_{s} \circ p_{s+1} \circ f_{s+1}=$ $p_{s} \circ f_{s}$ and with $A F\left(f_{s+1}\right) \geq A F\left(f_{s}\right)-\epsilon_{s}$.

Proof. Part (a) follows immediately from 3.1. The proofs of parts (b), (c) and (d) are similar: the image of $f_{s} \in \pi_{n}\left(S_{s}\right)$ under the map $S_{s} \stackrel{\iota \wedge 1}{\longrightarrow} \ell \wedge S_{s}$ produces a class $z_{s} \in \pi_{n}\left(\ell \wedge S_{s}\right)=E_{1}^{s, n+s}\left(\Sigma \mathbf{B}_{2 i+1}^{2 i+2 j} ; \ell\right)$ which is a $\delta_{1}$-cycle in the $\ell$-Adams spectral sequence for $\Sigma \mathbf{B}_{2 i+1}^{2 i+2 j}-\left(E_{1}\left(\Sigma \mathbf{B}_{2 i+1}^{2 i+2 j} ; \ell\right), \delta_{1}\right)$ stands for the first term in such spectral sequence. Since $A F\left(f_{s}\right)>0, z_{s}$ lies in fact in the $\ell$-regular complex $\mathcal{C}\left(\Sigma \mathbf{B}_{2 i+1}^{2 i+2 j}\right)$, and since this is concentrated in total degrees $t \equiv 0(\bmod q)$, we are forced to have $z_{s}=0$ if $n+s \not \equiv 0(\bmod q)$, in which case the required lifting $f_{s+1} \in$ $\pi_{n}\left(S_{s+1}\right)$ is given by 3.1. In general, the hypothesis will allow us to find a homotopy class $e_{s-1} \in \pi_{n+1}\left(\ell \wedge S_{s-1}\right)=E_{1}^{s-1, n+s}\left(\Sigma \mathbf{B}_{2 i+1}^{2 i+2 j} ; \ell\right)$ such that $\delta_{1}\left(e_{s-1}\right)=z_{s}$; that is, in the diagram 


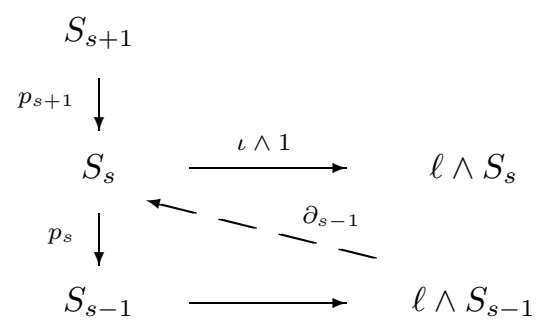

the relation $(i \wedge 1)_{*}\left(\partial_{s-1}\left(e_{s-1}\right)\right)=(i \wedge 1)_{*}\left(f_{s}\right)$ holds, where $\partial_{s-1}$ is the connecting map in the cofibration $S_{s} \stackrel{p_{s}}{\longrightarrow} S_{s-1} \rightarrow \ell \wedge S_{s-1}$ (details given below). In particular, the class $f_{s}^{\prime}=f_{s}-\partial_{s-1}\left(e_{s-1}\right)$ lifts to a homotopy class $f_{s+1} \in \pi_{n}\left(S_{s+1}\right)$ whose Adams filtration will be controlled by 3.1 and by Lemma 2.1 or Lemma 2.2, according to whether $s \geq 3$ or $s=2$ respectively. We give full details for the case $s \leq 2$ and $n+s=q m$.

When $s=1$, the remarks after (4) yield the commutative diagram with cofiber rows

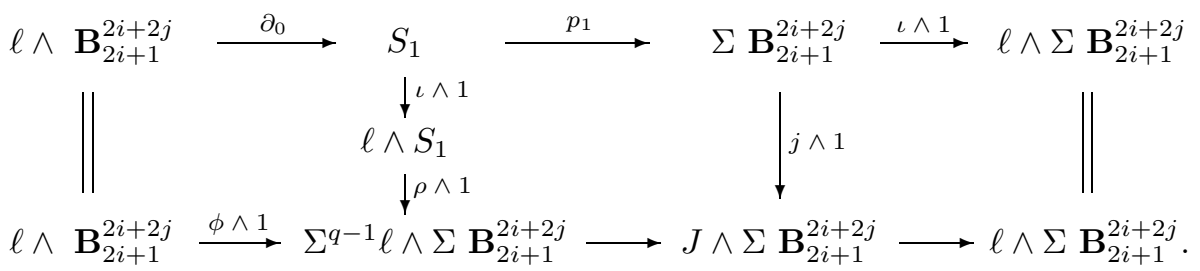

Therefore the present hypothesis together with 2.3 implies that, up to classes arising from split $\mathbb{F}_{p}$-Eilenberg Mac Lane spectra, $z_{1}$ is a $\delta_{1}$-boundary: $z_{1}=\delta_{1}\left(e_{0}\right)+\kappa$, where $e_{0} \in \pi_{n}\left(\ell \wedge \mathbf{B}_{2 i+1}^{2 i+2 j}\right)=\ell_{q m-1}\left(\mathbf{B}_{2 i+1}^{2 i+2 j}\right)$ and $\kappa$ is a homotopy class arising from the $\mathbb{F}_{p}$-Eilenberg Mac Lane split portion in $\ell \wedge S_{1}$. Since by hypothesis $m>i+j$, a standard Adams chart for $\ell_{*}\left(\mathbf{B}_{2 i+1}^{2 i+2 j}\right)$ (as the one in Figure 2) shows that every element in $\ell_{q m-1}\left(\mathbf{B}_{2 i+1}^{2 i+2 j}\right)$ has positive Adams filtration. In particular $\kappa$ must be trivial. It remains to control the Adams filtration of $e_{0}$. If $\nu(m) \geq j-1$, the operation $\pi_{q m-1}\left(\ell \wedge \mathbf{B}_{2 i+1}^{2 i+2 j}\right) \stackrel{\phi}{\rightarrow} \pi_{q(m-1)-1}\left(\ell \wedge \mathbf{B}_{2 i+1}^{2 i+2 j}\right)$ is trivial (see for instance the beginning of the proof for 2.3), that is, the first component of $z_{1}=\delta_{1}\left(e_{0}\right)$ is trivial, and in view of $[7$, Theorem $3.6(\mathrm{~b})]$, all other components of $z_{1}$ must also be trivial. Thus $z_{1}=0$ and we can take $e_{0}=0$. When $\nu(m)<j-1$, the first component of $z_{1}$ is either trivial (in which case $z_{1}=0$ as above) or has Adams filtration $A F\left(e_{0}\right)+\nu(m)$ in view of the initial observations in the proof of 2.3. In particular $A F\left(e_{0}\right)+\nu(m) \geq A F\left(z_{1}\right) \geq A F\left(f_{1}\right)$, so that the Adams filtration of the class $f_{1}^{\prime}$ in the preliminary remarks of this proof is at least $A F\left(f_{1}\right)-\nu(m)$ as required.

If $s=2$ let $r=\max \{1, t\}$, where $t$ stands for $\nu(m), 1$ or $\mu((p-1)(m+1-i))-1$, according to whether (ii), (iii) or (iv) of the hypothesis holds. The isomorphism in (3) implies that the cycle $z_{2} \in \mathcal{C}\left(\Sigma \mathbf{B}_{2 i+1}^{2 i+2 j}\right)=\mathcal{C}(i+j, i)$, being of Adams filtration at least $a=A F\left(f_{2}\right)$, lies in fact in $\mathcal{C}(i+j+a, i+a)$. From 2.2, $z_{2}$ produces a trivial homology class as an element in $\mathcal{C}(i+j+a-r, i+a-r)$; that is, there exists $e_{1} \in \mathcal{C}(i+j+a-r, i+a-r)$ such that $d\left(e_{1}\right)=z_{2}$, where $d$ denotes the differential in $\mathcal{C}(i+j+a-r, i+a-r)$. Observe in particular that the Adams 
filtration of $e_{1}$, as an element of $\mathcal{C}(i+j, i)$, is at least $a-r$. Although the natural inclusion $\mathcal{C}(i+j, i) \hookrightarrow E_{1}\left(\Sigma \mathbf{B}_{2 i+1}^{2 i+2 j} ; \ell\right)$ is not in general a map of complexes, it does preserve the differential of elements with positive Adams filtration (see for instance the remarks before [8, Proposition 3.4]); therefore we still have the required relation $\delta_{1}\left(e_{1}\right)=z_{2}$ in $E_{1}\left(\Sigma \mathbf{B}_{2 i+1}^{2 i+2 j} ; \ell\right)$, and the argument can be completed as indicated in the preliminary remarks of this proof.

With the same ideas but using 2.4 and 2.5 instead of 2.1,2.2 and 2.3, we can prove the following result, which although not complete, suffices for our applications.

Theorem 3.4. Let $\Sigma \mathbf{B}_{2 i}^{2 i+2 j} \stackrel{p_{1}}{\longleftarrow} S_{1} \stackrel{p_{2}}{\longleftarrow} S_{2} \stackrel{p_{3}}{\longleftarrow} \cdots$ be the standard $\ell$-resolution of $\Sigma \mathbf{B}_{2 i}^{2 i+2 j}$ and suppose given a map $S^{n} \stackrel{f_{s}}{\longrightarrow} S_{s}$, where $n=q m-1$ and $m>i+j$.

(a) In the case $s=0$ assume the composite $S^{n} \stackrel{f_{0}}{\longrightarrow} \Sigma \mathbf{B}_{2 i}^{2 i+2 j} \stackrel{\iota \wedge 1}{\longrightarrow} \ell \wedge \mathbf{B}_{2 i}^{2 i+2 j}$ is trivial; then $f_{0}$ can be lifted to a map $f_{1}: S^{n} \rightarrow S_{1}$ with $A F\left(f_{1}\right) \geq A F\left(f_{0}\right)-1$.

(b) In the case $s=1$ assume $A F\left(f_{1}\right) \geq 1$ and that the composite $S^{n} \stackrel{f_{1}}{\longrightarrow} S_{1} \stackrel{p_{1}}{\longrightarrow}$ $\Sigma \mathbf{B}_{2 i}^{2 i+2 j} \stackrel{j \wedge 1}{\longrightarrow} J \wedge \Sigma \mathbf{B}_{2 i}^{2 i+2 j}$ is trivial; then there is a map $S^{n} \stackrel{f_{2}}{\longrightarrow} S_{2}$ such that $p_{1} \circ p_{2} \circ f_{2}=p_{1} \circ f_{1}$ and with

(i) $A F\left(f_{2}\right) \geq A F\left(f_{1}\right)-1$ if $\nu(m) \geq j-1$.

(ii) $A F\left(f_{2}\right) \geq A F\left(f_{1}\right)-\nu(m)-1$ if $\nu(m)<j-1$.

(c) In the case $s=2$ assume $A F\left(f_{2}\right) \geq \nu(m-i)+1$; then there is a map $S^{n} \stackrel{f_{3}}{\rightarrow} S_{3}$ such that $p_{2} \circ p_{3} \circ f_{3}=p_{2} \circ f_{2}$ and $A F\left(f_{3}\right) \geq A F\left(f_{2}\right)-\nu(m-i)-1$.

(d) In the case $s \geq 3$ assume $A F\left(f_{s}\right) \geq \varepsilon_{s}$, where $\varepsilon_{s}=1$ if $n+s \not \equiv 0,1$ $(\bmod q)$ and $\varepsilon_{s}=2$ otherwise; then there is a map $S^{n} \stackrel{f_{s+1}}{\longrightarrow} S_{s+1}$ such that $p_{s} \circ p_{s+1} \circ f_{s+1}=p_{s} \circ f_{s}$ and with $A F\left(f_{s+1}\right) \geq A F\left(f_{s}\right)-\varepsilon_{s}$.

For a ring spectrum $E$, the $E$-filtration of a homotopy class $\alpha \in \pi_{*}(X)$ is defined as the largest integer $s$ so that $\alpha$ lifts to the $s$-stage in the standard $E$-resolution for $X$. In this notation, what we have called "Adams filtration" $(A F)$ corresponds to " $H \mathbb{F}_{p}$-filtration". The map $\ell \rightarrow H \mathbb{F}_{p}$ corresponding to the generator in $H^{0}\left(\ell, \mathbb{F}_{p}\right)$ yields a map from the standard $\ell$-resolution of any spectrum $X$ to the corresponding $H \mathbb{F}_{p}$-resolution of $X$. In particular, the $H \mathbb{F}_{p}$-filtration of any homotopy class is no less than the corresponding $\ell$-filtration. On the other hand, the results above imply that the $\ell$-filtration of a homotopy class with trivial $J$-Hurewicz image is almost as large as its $H \mathbb{F}_{p}$-filtration. The success in the application of these techniques to the study of homotopy classes depends on establishing suitable upper bounds for their $\ell$-filtration. For the sphere spectrum this is done in [8] through direct calculations. In general we use a variation of the standard $\ell$-resolution. The author learned of the following construction from Douglas C. Ravenel.

Pinching the bottom cell of the first two spectra in (4) produces a cofibration $\bar{J} \rightarrow \bar{\ell} \stackrel{\bar{\phi}}{\longrightarrow} \Sigma^{q} \ell$ fitting as the top row of the commutative diagram with cofiber rows and columns

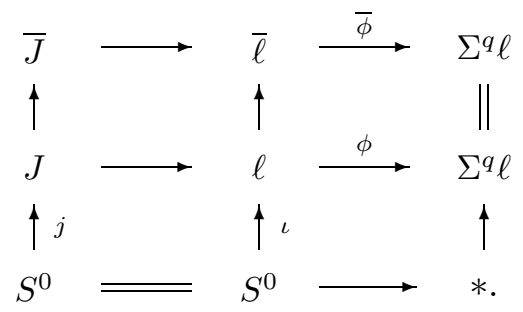


From the observations after (4), the map $\bar{\phi}: \bar{\ell} \rightarrow \Sigma^{q} \ell$ can be explicitly given as the composite $\bar{\ell} \stackrel{\iota \wedge 1}{\longrightarrow} \ell \wedge \bar{\ell} \stackrel{\rho}{\longrightarrow} \Sigma^{q} \ell$, so that for any map $f: X \rightarrow Y$ we can construct the commutative diagram

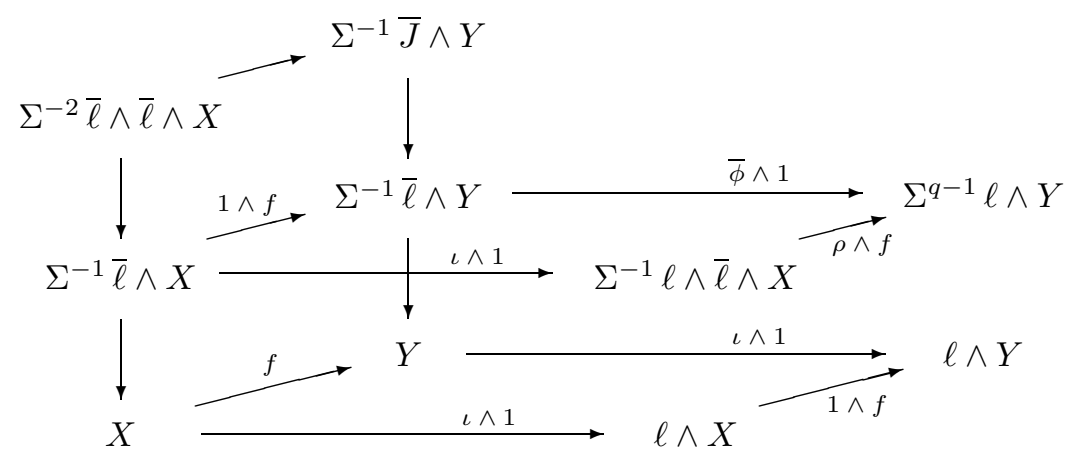

with cofiber sequences formed by a map going down followed by the corresponding horizontal right arrow. Repetition of this process starting with the identity $X \stackrel{1}{\longrightarrow}$ $X$ yields a map from the standard $\ell$-resolution of $X$ into the "resolution" $X \leftarrow$ $\Sigma^{-1} \bar{\ell} \wedge X \leftarrow \Sigma^{-1} \bar{J} \wedge X \leftarrow \Sigma^{-2} \bar{\ell} \wedge \bar{J} \wedge X \leftarrow \Sigma^{-2} \bar{J} \wedge \bar{J} \wedge X \leftarrow \cdots$ of $X$.

Proposition 3.5. Let $X \leftarrow S_{1} \leftarrow S_{2} \leftarrow \cdots$ be the standard $\ell$-resolution of a cconnected spectrum $X$. Then the composite $S_{s} \rightarrow S_{s-1} \rightarrow \cdots \rightarrow X$ factors through $a\left(s\left(p^{2}-p-1\right)-(p-1)(p-2) \iota_{s}+c\right)$-connected spectrum $R_{s}$, where $\iota_{s}=1$ if $s$ is odd and $\iota_{s}=0$ otherwise.

Proof. Let $X \leftarrow R_{1} \leftarrow R_{2} \leftarrow \cdots$ be the resolution of $X$ described above, so that $R_{2 s}=\Sigma^{-s} \bar{J}^{s} \wedge X$ and $R_{2 s+1}=\Sigma^{-1} \bar{\ell} \wedge R_{2 s}$ (here $\bar{J}^{s}$ stands for the $s$-fold iterated smash product of $\bar{J}$ with itself). By construction, the first nontrivial homotopy group of $\bar{\ell}$ appears in degree $q$. On the other hand, since the first element not in the image of $J$ lies in degree $p q-2$, the first nontrivial homotopy group of $\bar{J}$ appears in degree $p q-1$. The result follows from the above considerations.

\section{J-OBSTRUCtions}

Problem 1.5 posed in the introduction can be solved in a relatively easy way if instead of asking for essential maps, we ask for maps with nontrivial Hurewicz image under the $J$ spectrum. The next two propositions give a complete answer to this $J$-version of Problem 1.5. The proofs are straightforward calculations with the $J$ spectrum and will be omitted.

Proposition 4.1. Let $i, j$ be positive integers.

(a) For any $l$ the collapsing map $\Sigma \mathbf{B}_{2 i}^{2 i+2 l} \rightarrow \Sigma \mathbf{B}_{2 i+1}^{2 i+2 l}$ induces isomorphisms in $J$-homology groups in dimensions congruent with $-1 \bmod q$.

(b) If $j \leq \nu(i+j+1)$, then for any $l, \theta_{l}$ has nontrivial $J$-Hurewicz image.

(c) If $\nu(i+j+1)<j$, then the largest integer $l$ such that $\theta_{l}$ has nontrivial $J$-Hurewicz image is $l=\nu(i+j+1)+1$.

Proposition 4.2. Let $i, j$ be positive integers.

(a) For any $l$ the collapsing map $\Sigma \mathbf{B}_{2 i}^{2 i+2 l+1} \rightarrow \Sigma \mathbf{B}_{2 i+1}^{2 i+2 l+1}$ induces isomorphisms in $J$-homology groups in dimensions congruent with $-1 \bmod q$. 
(b) If $j \leq \nu(i+j+1)$, then for any $l$, $\vartheta_{l}$ has nontrivial $J$-Hurewicz image.

(c) If $\nu(i+j+1)<j$, then the largest integer $l$ such that $\vartheta_{l}$ has nontrivial $J$-Hurewicz image is given by

$$
l= \begin{cases}\nu(i+j+1) & \text { if } \quad \nu(i+j+1)=j-1 \\ \nu(i+j+1)+1 & \text { if } \quad \nu(i+j+1)<j-1 .\end{cases}
$$

The obstructions arising in the classification of stunted lens spectra [9] have the form $\bar{\theta}_{j, i, j}$ and $\bar{\vartheta}_{j, i, j}$. These maps turn out to be trivial whenever they have trivial $J$-Hurewicz image as the following result shows.

Proposition 4.3. Let $i$ and $j$ be positive integers,

(a) $\bar{\theta}_{j}$ is stably null homotopic if and only if $\nu(i+j+1)<j-1$.

(b) $\bar{\vartheta}_{j}$ is stably null homotopic if and only if $\nu(i+j+1)<j$.

Remark 4.4. This proposition solves Problem 1.5 for the maps $\bar{\theta}_{l}$ and $\bar{\vartheta}_{l}$ in the case $\nu(i+j+1)=j-1$. As we will see in the next section, this is the case where $\ell$-resolutions techniques give the least information.

Proof. By Propositions 4.1 and 4.2, both conditions are necessary; moreover the sufficiency in (b) is a restatement of [9, Corollary 3.5]. Finally if $\nu(i+j+1)<j-1$ but $\bar{\theta}_{j}$ is essential, then $\bar{\theta}_{j}$ is up to a unit the attaching map for the top cell of $\Sigma \mathbf{B}_{2 i+1}^{2 i+2 j+1}$. In particular $\Sigma \mathbf{B}_{2 i+2}^{2 i+2 j+1}$ is S-reducible, which by [9, Lemma 6.1] implies $\nu(i+j+1) \geq j-1$, a contradiction.

A consequence of 4.3 is the following well known result.

Corollary 4.5. Let $\pi: S^{(i+j+1) q-1} \rightarrow \Sigma \mathbf{B}_{2 i+1}^{2 i+2 j}$ be the attaching map for the top cell in $\Sigma \mathbf{B}_{2 i+1}^{2 i+2 j+1}$. If $\pi$ is essential and becomes null homotopic by pinching the bottom cell in $\Sigma \mathbf{B}_{2 i+1}^{2 i+2 j}$, then $\pi$ agrees, up to a unit with $\bar{\theta}_{j}$.

Since the $p$-localization of $\pi_{t q-2}\left(S^{0}\right)$ is trivial for $t<p$, we also have

Corollary 4.6. Fix integers $i$ and $j$ with $j<p-1$. Then

(a) $\theta_{j}$ is stably null homotopic if and only if $\nu(i+j+1)<j-1$.

(b) $\vartheta_{j}$ is stably null homotopic if and only if $\nu(i+j+1)<j$.

\section{Classification of stunted B spectra}

Using $J$-homology groups we have identified nontrivial maps in 1.5. Next we show that a number of the remaining maps are indeed null homotopic. In order to motivate the techniques, we start by proving the following mild improvement of $[8$, Theorem 3.9].

Theorem 5.1. Let $\alpha \in \pi_{n}\left(S^{0}\right)$ be in the kernel of the J-Hurewicz homomorphism. If the classical Adams filtration of $\alpha$ is at least $3+\varepsilon_{n}+\frac{(q+1) n}{q\left(p^{2}-p-1\right)}$, then $\alpha=0$. Here $\varepsilon_{n}=0$ if $n+2 \not \equiv 0(\bmod q)$ and $\varepsilon_{n}=\nu(n+2)$ otherwise.

Proof. Let $S^{0} \leftarrow S_{1} \leftarrow S_{2} \leftarrow \cdots$ be the standard $\ell$-resolution for the sphere spectrum. By 3.5 the Adams projection $S_{s} \rightarrow S^{0}$ factors through a spectrum $R_{s}$ whose first nontrivial homotopy group appears in dimension at least $s\left(p^{2}-p-1\right)-(p-1)(p-2)$. Therefore we will be done once we find a lifting of $\alpha$ to $S_{M}$ for $M=\left[\frac{n+(p-1)(p-2)}{p^{2}-p-1}\right]+1$. Parts (a), (b) and (c) of 3.2 provide a lifting $f_{3}$ to $S_{3}$ with Adams filtration at least $\psi=A F(\alpha)-\varepsilon_{n}-3$. Thus we have to 
(perhaps modify and) lift $M-3$ times more. By $3.2(\mathrm{~d})$, this is possible provided $\psi \geq a(q+1)+b+1$, where $M-3=a q+b$ and $0 \leq b<q$. Straightforward algebraic manipulations show that such condition is implied by the hypothesis.

Theorem 5.1 sets upper bounds on the classical Adams filtration of nontrivial classes $\alpha \in \pi_{*}\left(S^{0}\right)$ with trivial $J$-Hurewicz image. The analogous result for stunted B spaces turns out to be a suitable tool in the study of Problem 1.5. Indeed, as we show below, most of the classes in 1.2 with trivial $J$-Hurewicz image, are in fact null homotopic because of their "high" Adams filtration. The next two results can be slightly improved in particular cases by using the full strength of the results in Section 3. This is done for instance in 5.8 to complete the classification of stunted B spectra for $p=11$.

Theorem 5.2. If $i$ and $j$ are positive integers satisfying

$$
j-\nu(i+j+1)-\nu(j)-2 \geq \frac{(j-1)(q+1)}{p^{2}-p-1},
$$

then $\bar{\theta}_{l}$ is stably null homotopic if and only if it has trivial J-Hurewicz image.

Proof. Let $l=\nu(i+j+1)+2$; by 4.1 it suffices to show that $\bar{\theta}_{l}$ is null homotopic. Let $\cdots \rightarrow S_{2} \rightarrow S_{1} \rightarrow \Sigma \mathbf{B}_{2 i+1}^{2 i+2 l}$ be the standard $\ell$-resolution for $\Sigma \mathbf{B}_{2 i+1}^{2 i+2 l}$. By 3.5 the Adams projection $S_{s} \rightarrow \Sigma \mathbf{B}_{2 i+1}^{2 i+2 l}$ factors through a spectrum $R_{s}$ satisfying $\pi_{r}\left(R_{s}\right)=0$ for $r<(i+1) q+s\left(p^{2}-p-1\right)-(p-1)(p-2)$, so that we will be done once we find a lifting of $\bar{\theta}_{l}$ to $S_{M}$ where $M=\left[\frac{j q-1+(p-1)(p-2)}{p^{2}-p-1}\right]+1=$ $\left[\frac{q(j-1)}{p^{2}-p-1}\right]+2$. Such a lifting is provided by Theorem 3.3. Indeed, it is well known that $A F\left(\bar{\alpha}_{j}\right)=j-\nu(j)$, so we get a lifting of $\bar{\theta}_{l}$ to $S_{2}$ with Adams filtration at least $\psi=j-\nu(i+j+1)-\nu(j)-2$. Then $f_{2}$ needs to be (perhaps modified and) lifted $M-2$ times more, which by 3.3 can be done provided $\psi \geq a(q+1)+b$, where $M-2=a q+b$ and $0 \leq b<q$. As in 5.1, this is implied by the hypothesis.

Likewise, Theorem 3.4 gives

Theorem 5.3. If $i$ and $j$ are positive integers satisfying

$$
j-\nu(i+j+1)-\nu(j)-\nu(j+1)-3 \geq \frac{j(q+2)}{p^{2}-p-1},
$$

then $\theta_{l}$ is stably trivial if and only if it has trivial J-Hurewicz image.

The following corollary should be compared with 4.5 .

Corollary 5.4. Let $l=\nu(i+j+1)+1$ and suppose $l<j$. If the conclusion of 5.3 is true, then the stable class of $\vartheta_{l}$ factors as

$$
S^{(i+j+1) q-1} \stackrel{\alpha}{\longrightarrow} S^{(i+l+1) q} \stackrel{\pi}{\longrightarrow} \Sigma \mathbf{B}_{2 i}^{2 i+2 l+1},
$$

where $\pi$ is the attaching map for the top cell in $\mathbf{B}_{2 i}^{2 i+2 l+2}$ and $\alpha$ is a class of order $p$ in the stem $(j-l) q-1$ with nontrivial J-Hurewicz image.

Proof. By hypothesis we get such a factorization and by $4.2 \alpha$ must be detected by the $J$ spectrum. Moreover since $S^{(i+j+1) q-1} \stackrel{\vartheta_{l}}{\longrightarrow} \Sigma \mathbf{B}_{2 i}^{2 i+2 l+1} \stackrel{\text { pinch }}{\longrightarrow} S^{(i+l+1) q}$ is null homotopic and $S^{(i+l+1) q} \stackrel{\pi}{\longrightarrow} \Sigma \mathbf{B}_{2 i}^{2 i+2 l+1} \stackrel{\text { pinch }}{\longrightarrow} S^{(i+l+1) q}$ is the degree $p$ map, it follows that $p \alpha=0$. 
Remark 5.5. By 4.1 and 4.2 , the conclusions in 5.2 and 5.3 are certainly true provided $j \leq \nu(i+j+1)$. On the other hand, the hypothesis in the last two theorems require not only $j-\nu(i+j+1)$ to be positive, but "sufficiently large", roughly $j-\nu(i+j+1)>\frac{(j-1)(q+1)}{p^{2}-p-1}+1$, in the case of 5.2. Fixing $j$ and letting $i$ vary, we see that the fraction of cases missed by this restriction is roughly $\left(\frac{1}{p}\right)^{m}$, where $m=\frac{(j-1) p(p-3)}{p^{2}-p-1}$. This intends to give a more precise meaning to the expression "...in a wide range of cases ...", used after 1.5 in the introduction (note however that the results are rather weak for $p=3$ ). On the other hand, these technicalities are the reason why we do not attempt to obtain a version of 5.2 or 5.3 for maps $\bar{\vartheta}_{l}$ or $\vartheta_{l}$. Indeed, if $\ell$-resolution techniques were to prove the triviality of $\vartheta_{l}$ say, one would need some sort of restriction on $j-\nu(i+j+1)$ being "large"; however, if $\theta_{l}$ itself were not already trivial, 4.2 would suggest that $j-\nu(i+j+1)=1$ (and $l=j$ ). Despite this, we can still prove the triviality of a number of classes $\vartheta_{j, i, j}$, even when $j-\nu(i+j+1)=1$ (and in general when $j-\nu(i+j+1$ ) is "small enough"). The methods are rather geometrical and more accessible than those involving $\ell$-resolutions (see for instance [9]).

Proposition 5.6. If $\nu(i+j+1)<j \leq 2 \nu(i+j+1)$, then $\vartheta_{j, i, j}$ is stably trivial.

Proof. The stable dual of $\mathbf{B}_{2 i}^{2 i+2 j+1}$ is $\mathbf{B}_{-2 i-2 j-2}^{-2 i-1}$ in view of [9, Lemma 6.1]. Thus by duality we must show the triviality of the composite $\mathbf{B}_{2 a}^{2 a+2 s+1} \stackrel{\pi^{\prime}}{\longrightarrow}$ $S^{(a+s) q} \stackrel{\bar{\alpha}_{s}}{\longrightarrow} S^{a q+1}$ for $\nu(a)<s \leq 2 \nu(a)$, where $\pi^{\prime}$ is the composition of the collapsing map $\mathbf{B}_{2 a}^{2 a+2 s+1} \rightarrow \mathbf{B}_{2 a+2 s}^{2 a+2 s+1}=S^{(a+s) q} \vee S^{(a+s+1) q-1}$ and the wedge projection $S^{(a+s) q} \vee S^{(a+s+1) q-1} \rightarrow S^{(a+s) q}$. In [3, Section 2] a complex $p-1$ dimensional vector bundle $\beta$ over $\mathbf{B}=\mathbf{B}_{1}^{\infty}$ is described, with the property that the Thom complex $\left(\mathbf{B}^{2 s+1}\right)^{a \beta}$ of the $a$-multiple of $\beta$ over $\mathbf{B}^{2 s+1}$ is homeomorphic to the stunted space $\mathbf{B}_{2 a}^{2 a+2 s+1}$. The realification of $\beta$ has $K O$-order $p^{s}$ over $\mathbf{B}^{2 s}$ and $K O$-order $p^{s-1}$ over $B^{2 s-1}$. Since $a \beta$ restricts trivially to $B^{2 \nu(a)}$, there is a bundle $\mu: \mathbf{B}_{2 \nu(a)+1}^{2 s+1} \rightarrow B O$ inducing $a \beta$ under the collapsing map $\mathbf{B}^{2 s+1} \rightarrow \mathbf{B}_{2 \nu(a)+1}^{2 s+1}$. This map induces a monomorphism in $K O$-theory, so that $\mu^{\prime}=p^{d} \mu$ is a nontrivial bundle over $\mathbf{B}_{2 \nu(a)+1}^{2 s+1}$ of $K O$-order $p(d=s-\nu(a)-1)$ inducing $p^{d} a \beta$ under the collapsing $\mathbf{B}^{2 s+1} \rightarrow \mathbf{B}_{2 \nu(a)+1}^{2 s+1}$. Since $p^{d} a \beta$ is trivial over $\mathbf{B}^{2 s-1}$ and the collapsing $\mathbf{B}^{2 s-1} \rightarrow \mathbf{B}_{2 \nu(a)+1}^{2 s-1}$ is injective in $K O$-theory, it follows that $\mu^{\prime}$ induces a trivial bundle under the inclusion $\mathbf{B}_{2 \nu(a)+1}^{2 s-1} \hookrightarrow \mathbf{B}_{2 \nu(a)+1}^{2 s+1}$. Thus there is a bundle $\omega: \mathbf{B}_{2 s}^{2 s+1} \rightarrow B O$ inducing $\mu^{\prime}$ under the collapsing $\mathbf{B}_{2 \nu(a)+1}^{2 s+1} \rightarrow \mathbf{B}_{2 s}^{2 s+1}$. Since $\mathbf{B}_{2 s}^{2 s+1}=S^{s q} \vee S^{(s+1) q-1}$ and $\pi_{(s+1) q-1}(B O)=0, \omega$ must be induced in turn by a bundle $\omega^{\prime}: S^{s q} \rightarrow B O$ under the wedge projection $\mathbf{B}_{2 s}^{2 s+1} \rightarrow S^{s q}$. It results in a commutative diagram:

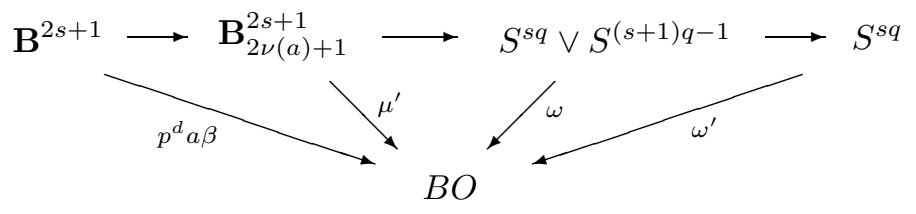

Observe furthermore that since $\mu^{\prime}$ has $K O$-order $p, \omega^{\prime}$ must be a multiple prime to $p$ of the generator in $\pi_{s q}(B O)=\mathbb{Z}$. Denoting by $X^{\alpha}$ the Thom complex of the 
bundle $\alpha$ over $X$, and by $\overline{X^{\alpha}}$ the cofiber of the inclusion $S^{0} \hookrightarrow X^{\alpha}$ (all Thom complexes will be treated as spectra with zero dimensional bottom Thom cell), we have a commutative diagram:

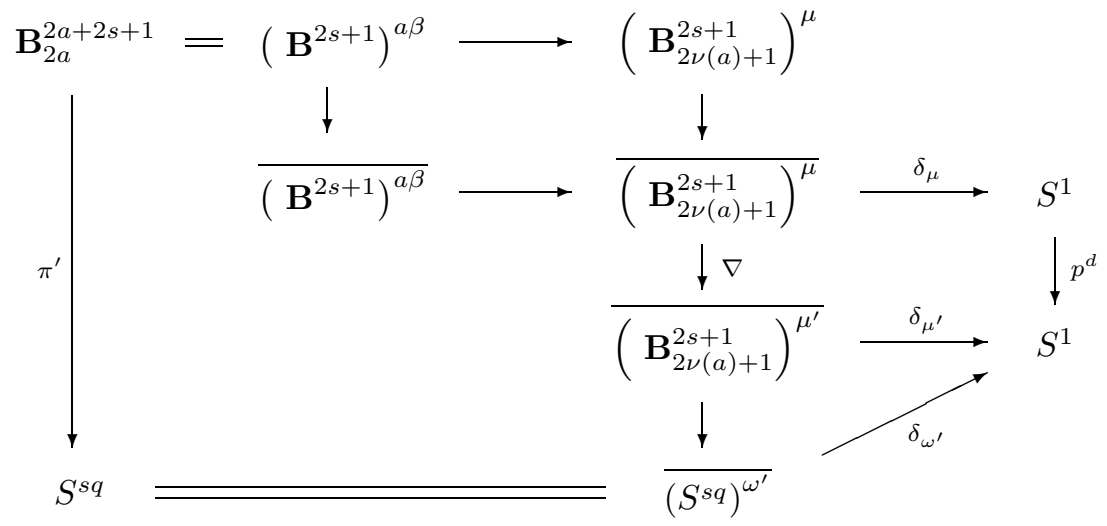

Here the unnamed maps are induced either by pinching the bottom Thom cell or by Thomifying maps between vector bundles; moreover a map $\delta_{\alpha}: \overline{X^{\alpha}} \rightarrow S^{1}$ is defined through the Puppe cofiber sequence $S^{0} \hookrightarrow X^{\alpha} \rightarrow \overline{X^{\alpha}} \stackrel{\delta_{\alpha}}{\longrightarrow} S^{1}$. The hypothesis $\nu(a)<s \leq 2 \nu(a)$ implies that, as a space, $\mathbf{B}_{2 \nu(a)+1}^{2 s+1}$ is a double suspension. By [6, Proposition 3.3 and Corollary 3.4], both $\overline{\left(\mathbf{B}_{2 \nu(a)+1}^{2 s+1}\right)^{\mu}}$ and $\overline{\left(\mathbf{B}_{2 \nu(a)+1}^{2 s+1}\right)^{\mu^{\prime}}}$ are homeomorphic to the suspension spectrum of $\mathbf{B}_{2 \nu(a)+1}^{2 s+1}$, with a homeomorphism $\nabla: \overline{\left(\mathbf{B}_{2 \nu(a)+1}^{2 s+1}\right)^{\mu}} \simeq \overline{\left(\mathbf{B}_{2 \nu(a)+1}^{2 s+1}\right)^{\mu^{\prime}}}$ fitting in the above diagram. As observed above, the $p$-localization of $\omega^{\prime}$ must be a generator of $\pi_{s q}(B O)_{(p)}=\mathbb{Z}_{(p)}$, so that $\delta_{\omega^{\prime}}$ (or a suitable multiple of it) generates the $p$-component of the image of the classical $J$-homomorphism in the $s q-1$ stem. The result follows by observing that the composition of the last two maps in $\left(\mathbf{B}^{2 s+1}\right)^{a \beta} \rightarrow \overline{\left(\mathbf{B}^{2 s+1}\right)^{a \beta}} \rightarrow \overline{\left(\mathbf{B}_{2 \nu(a)+1}^{2 s+1}\right)^{\mu}} \stackrel{\delta_{\mu}}{\longrightarrow}$ $S^{1}$ agrees with the corresponding connecting map $\overline{\left(\mathbf{B}^{2 s+1}\right)^{a \beta}} \stackrel{\delta_{a \beta}}{\longrightarrow} S^{1}$.

We now apply these results to the homotopy classification of stunted $\mathbf{B}$ spectra. We begin by proving the following more detailed version of Proposition 1.4.

Proposition 5.7. Let $\mathcal{S}$ be the set consisting of the spectra in (1) of the introduction and their duals. Assume that $\vartheta_{j, r, j}: S^{(r+j+1) q-1} \rightarrow \Sigma \mathbf{B}_{2 r}^{2 r+2 j+1}$ is trivial whenever $B_{2 r}^{2 r+2 j+1}$ is non-reducible and lies in $\mathcal{S}$. Then the spectra in (1) which are neither reducible nor coreducible are all equivalent.

Proof. Let $\mathbf{B}_{2 i+2 t}^{2 i+2 t+2 j+1}$ and $\mathbf{B}_{2 i}^{2 i+2 j+1}$ be neither reducible nor coreducible ( $t$ as in (1)). By 1.3 we may assume that one of the following maps is stably essential:

$$
\begin{aligned}
\theta_{j, i, j}: S^{(i+j+1) q-1} & \rightarrow \Sigma \mathbf{B}_{2 i}^{2 i+2 j} \\
\theta_{j,-i-j-t-1, j}: S^{-(i+t) q-1} & \rightarrow \Sigma \mathbf{B}_{-2 i-2 t-2 j-2}^{-2 i-2 t-2} .
\end{aligned}
$$

Say (9) is essential. Since $\Sigma \mathbf{B}_{2 i}^{2 i+2 j+1}$ is non-reducible, $\theta_{j, i, j}$ agrees up to a unit with the attaching map $\pi: S^{(i+j+1) q-1} \rightarrow \Sigma \mathbf{B}_{2 i}^{2 i+2 j}$ for the top cell of $\Sigma \mathbf{B}_{2 i}^{2 i+2 j+1}$. On the other hand, by [9, Theorem 4.5] we have a stable equivalence $\Sigma \mathbf{B}_{2 i}^{2 i+2 j} \stackrel{h}{\simeq}$ 
$\Sigma^{1-t q} \mathbf{B}_{2 i+2 t}^{2 i+2 j+2 t}$. Let $h^{\prime}$ be the restriction to the $(i+1) q$ dimensional skeleton. Since $\bar{\alpha}_{1}$ and $\bar{\alpha}_{j}$ compose trivially, the composite

$$
S^{(i+j+1) q-1} \stackrel{\bar{\alpha}_{j}}{\longrightarrow} S^{(i+1) q} \hookrightarrow S^{i q+1} \vee S^{(i+1) q} \stackrel{h^{\prime}}{\longrightarrow} S^{i q+1} \vee S^{(i+1) q} \rightarrow S^{i q+1}
$$

is trivial (all spheres are localized at $p$ ) and we get a commutative diagram

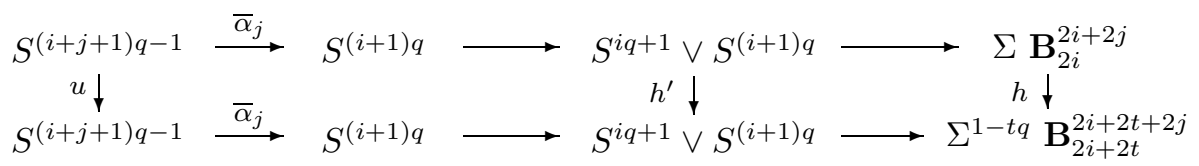

where $u$ is an equivalence. In particular $\theta_{j, i+t, j}$, being the bottom row of the diagram, is essential and as above, agrees up to a unit with the attaching map $\pi^{\prime}: S^{(i+j+t+1) q-1} \rightarrow \Sigma \mathbf{B}_{2 i+2 t}^{2 i+2 j+2 t}$ for the top cell of $\Sigma \mathbf{B}_{2 i+2 t}^{2 i+2 t+2 j+1}$. There results a commutative diagram

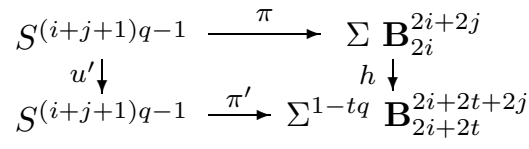

with $u^{\prime}$ an equivalence. The map between cofibers is the required equivalence. When (10) is essential, a similar argument produces an equivalence between the dual spectra.

Remark 5.8. By the results in [9], the only missing cases in the homotopy classification of stunted $\mathbf{B}$ spectra are those contained in Proposition 1.4. The methods in this paper allow us to show that the hypothesis in 1.4 does hold for $p \geq 7$, except perhaps for $p=7, j=7$ and $\nu(i+j+1)=3$. We finish the paper by indicating the way one can check the above assertion. By 4.6,5.3 and 5.6, the homotopy class $\vartheta_{j, i, j}: S^{(i+j+1) q-1} \rightarrow \Sigma \mathbf{B}_{2 i}^{2 i+2 j+1}$ is stably trivial if $\nu(i+j+1)<j$ and either one of the following holds:

a) $j<p-1$,

b) $\frac{j-1}{2}<\nu(i+j+1)$,

c) $\nu(i+j+1) \leq j-\nu(j)-\nu(j+1)-3-\frac{2 p j}{p^{2}-p-1}$.

Assume $p \geq 13$ and let $f_{p}(x)=x-\log _{p}(x)-\log _{p}(x+1)-3-\frac{2 p x}{p^{2}-p-1}$, so that $f_{p}(j)$ is less than or equal to the expression on the right of (c). Elementary manipulations show that $\frac{j-1}{2} \leq f_{p}(j)$ for $j \geq p+9$, whereas a simple inspection gives $\frac{j-1}{2} \leq$ $j-\nu(j)-\nu(j+1)-3-\frac{2 p j}{p^{2}-p-1}$ for $p-1 \leq j \leq p+8$. In any case either one of a), b) or c) holds. For $p=11$ similar techniques show that either one of b) or c) holds if $j \geq 12$. The triviality of the class $\vartheta_{j, i, j}$ for $j=10$ and $j=11$ is set by combining the full strength of 3.4 with 3.5. The situation for $p=7$ is treated similarly, giving the case $j=7$ and $\nu(i+j+1)=3$ as the only possible exception for the triviality of $\vartheta_{j, i, j}$. 


\section{REFERENCES}

[1] J. F. Adams. Lectures in generalised cohomology. Lecture Notes in Math., 99:1-138, 1969. MR 40:4943

[2] S. Araki. Typical formal groups in complex cobordism and $K$-theory. Lectures in Math., Kyoto Univ., Kinokuniya, 6, 1973. MR 51:1159

[3] D. M. Davis. Odd primary bo-resolutions and $K$-theory localization. Illinois J. Math., 30(1):79-100, 1986. MR 87g:55026

[4] D. M. Davis and M. E. Mahowald. Classification of the stable homotopy types of stunted real projective spaces. Pacific J. Math., 125(2):335-345, 1986. MR 88a:55006

[5] D. M. Davis and M. E. Mahowald. The image of the stable J-homomorphism. Topology, 28(1):39-58, 1989. MR 90c:55013

[6] S. Feder, S. Gitler, and K. Y. Lam. Composition properties of projective homotopy classes. Pacific J. Math., 68(1):47-61, 1977. MR 58:24263

[7] J. González. The regular complex in the $B P\langle 1\rangle$ Adams spectral sequence. Trans. Amer. Math. Soc. 350 (1998), 2629-2664. CMP 98:11

[8] J. González. A vanishing line in the $B P\langle 1\rangle$ Adams spectral sequence. To appear in Topology.

[9] J. González. Classification of the stable homotopy types of stunted lens spaces for an odd prime. Pacific J. Math., 176(2):325-343, 1996. MR 97m:55011

[10] D. C. Johnson and W. S. Wilson. Projective dimension and Brown-Peterson homology. Topology, 12:327-353, 1973. MR 48:12576

[11] R. M. Kane. Operations in connective K-theory. Mem. Amer. Math. Soc., 34(254), 1981. MR 82m:55025

[12] W. Lellmann. Operations and co-operations in odd-primary connective $K$-theory. J. London Math. Soc. (2), 29(3):562-576, 1984. MR 88a:55020

[13] W. Lellmann and M. E. Mahowald. The bo-Adams spectral sequence. Trans. Amer. Math. Soc., 300(2):593-623, 1987. MR 88c:55023

[14] R. Thompson. The $v_{1}$-periodic homotopy groups of an unstable sphere at odd primes. Trans. Amer. Math. Soc., 319(2):535-559, 1990. MR 90j:55021

Departamento de Matemáticas, Cinvestav, AP 14-740, México DF 07000

E-mail address: jesus@math.cinvestav.mx 